\title{
An Adaptive Eigenfunction Basis Strategy to Reduce Design Dimension in Topology Optimization
}

\section{Clay Sanders $^{1 \dagger} \quad \mid$ Marc Bonnet ${ }^{2 *} \quad$ Wilkins Aquino ${ }^{1 *}$}

${ }^{1}$ Department of Civil \& Environmental Engineering, Duke University, Durham, NC, 27708, USA

${ }^{2}$ POEMS (CNRS-ENSTA-INRIA), ENSTA

Paris, Palaiseau, France

\section{Correspondence}

Clay Sanders, Department of Civil \&

Environmental Engineering, Duke

University, Durham, NC, 27708, USA

Email: clay.sanders@duke.edu

\section{Present address}

$\dagger$ Department of Civil \& Environmental Engineering, Duke University, Durham, NC, 27708, USA

\section{Funding information}

U.S. Department of Energy Computational Science Graduate Fellowship:

DE-FG02-97ER25308
The concept of adaptive eigenspace basis (AEB) has recently proved effective for solving medium imaging problems. In this work, we present an AEB strategy for design parameterization in topology optimization (TO) problems. We seek the density design field as a linear combination of eigenfunctions, computed for an elliptic operator defined over the structural domain, and solve for the associated eigenfunction coefficients. Restriction to this truncated eigenspace drastically reduces the design dimension and imposes implicit regularization upon the solution, removing the need for auxiliary filtering operations and design-variable bound constraints. We furthermore develop the basis adaptation scheme inherent in the $A E B$, which iteratively recomputes the eigenfunction basis to conform to the evolving density field, enabling further dimension reduction and acceleration of the optimization process. The known aptitude of the adapted eigenfunctions to approximate piecewise constant fields is especially useful for TO as relevant design subspaces can be given lowdimensional representations. We propose criteria for the selection of the basis dimension and demonstrate the use of basis function selection as means for length scale control. We compare performance of the AEB against conventional TO implementations in problems for static linearelasticity, showing comparable structural solutions, computational cost benefits, and consistent design dimension reduction.

\section{KEYWORDS}

Adaptive eigenspace basis, Topology design, Dimensionality reduction

Abbreviations: AEB, adaptive eigenspace basis; TO, topology optimization; $\mathrm{EB}$, eigenspace basis. 


\section{1 | INTRODUCTION}

The aim of this paper is to formulate an effective design dimensionality reduction scheme for density-based topology optimization (TO), in which the spatial distribution of material is described by means of a "fictitious density" field. Conventional TO methods seek this density in a high-dimensional design space, such as that of all element-wise constant densities. By contrast, we propose to apply to TO problems an adaptive eigenspace basis (AEB) strategy, where density fields are represented using an adaptively-constructed basis of eigenfunctions defined over the structural domain. In this approach, the dimension of the design space is sharply reduced, as designs are adequately parameterized as linear combinations of a moderate number of density modes. We find that the truncated eigenfunction basis representation may substitute conventional methods to regularize the design space, such as filtering operations applied to the underlying fictitious-density design field. Additionally, we propose a series of adaptive eigenfunction basis updates, allowing the design field to capture the evolving design in an increasingly compressed form. Structural designs obtained with the AEB method, compared to conventional density-based methods, achieve comparable structural performance while enjoying benefits associated with a lower-dimensional optimization problem. We assert that our reduced-basis design approach offers attractive computational properties, associated with lower dimensional-designs and the elimination of conventional filters, and is amenable to TO schemes for a variety of physics applications.

The conventional approach to the formulation of TO problems is to represent the structural design using a spatially-varying "fictitious density" field, taking values in $(0,1)$ and representing interpolation between two material phases, or between solid and void. In discrete implementations, a vector of variables corresponding to element or nodal density values parameterizes the design. Penalization of intermediate density values encourages the allocation of full-density in advantageous regions of the structural domain [1]. Filtering operations are necessarily employed to regularize the density field and ensure solution existence; they are typically constructed as averaging kernel operations precluding high-frequency or mesh-dependent variation in the solution [2] or as diffusion-type PDEs [3].

The expense of solving the topology optimization problem naturally increases as the computational domain grows in dimension; large or finely-discretized domains not only require expensive state-space (displacement) solutions, but also feature design-space dimensions that grow in proportion to the state-space dimension. Strategies that reduce the dimension of the optimization problem, while incurring only slight effects upon solution quality, would offer attractive means to mitigate computational cost. In general, reduced-dimension representations of the design space may enable convergence in fewer iterations with improved stability. Second-order implementations involving the Hessian (or approximation of the Hessian), for instance, will have greatly reduced dimension and can enjoy improved convergence rates. In this work, we will address reduction of the design space dimension as means of improving the computational efficiency of solving the topology optimization problem.

Design space dimensionality reduction in topology optimization, though not widely studied, has previously been performed through various adaptive schemes. Guest et al. proposed an adaptive formulation of a "Heaviside projection method", which used a material indicator function to turn on and off design variables, in an adaptive manner, inside moving Heaviside radial projection regions [4]. The approach adaptively reduced the design space dimension by isolating the design variables subject to changes in value, principally those along the structural domain boundary. However, computational savings are limited by the non-smooth nature of the Heaviside projection and the use of genetic algorithms, which can incur heavy cost through numerous objective function evaluations.

In a similar vein, adaptive mesh refinement in topology optimization is a useful strategy to refine the evolving structural-void boundary and thereby allocate computational expense efficiently. In these strategies, goal-oriented error estimators can indicate regions of high solution or geometry interpolation error, identifying these regions as candidates for mesh refinement, while regions with low error may be coarsened without impact to solution error $[5,6]$. 
Multiple field solutions, in which separate meshes model the state and design fields, further reduce the dimension design represented by the adaptive meshes [7]. These methods, however, still require filtering operations to avoid development of mesh-dependent solutions and checkerboard patterns and retain high-dimensional design spaces for highly-refined structural domains.

Inverse medium problems, which aim at reconstructing unknown spatially-varying material coefficients, face similar computational challenges due to the potentially high dimension of the medium approximation space. Adaptive eigenspace basis (AEB) approaches have recently been formulated for elastodynamic [8, 9] or electromagnetic [10] medium inverse problems. They consist in expanding unknown coefficients upon a basis of eigenfunctions of an elliptic operator defined over the domain. Moreover, eigenfunctions are computed in an adaptive manner so that their spatial variation is concentrated in regions where coefficients have large gradients. They lead to medium approximation spaces of moderate dimension (typically 10-100) while allowing accurate medium reconstructions without reliance on stringent prior information. In this work, we therefore propose to exploit the AEB approach in TO problems. In this approach, the density is confined to the span of a basis of eigenfunctions of an elliptic operator defined over the structural domain, and the design variables become the coefficients of eigenfunction expansions, enabling a significant reduction in the dimensionality of the design space. Truncating bases by retaining low-frequency eigenfunctions also serves as a form of implicit regularization upon the design field, preventing the evolution of mesh-dependent, oscillating solutions; thus, the filtering operations typically required to control for mesh-dependence and checkerboarding become unnecessary. Following the adaptive framework of $[9,10]$, the eigenfunction basis is progressively adjusted to the developing density-field solution. This process rests on a sequence of optimization problems, where we compute a design solution, use that solution to define an adapted version of the elliptic operator for the eigenvalue problem, then compute a new eigenbasis. As the eigenfunctions of these operators are constructed to conform to the developing solution, and in particular are shown [11] to represent piecewise-constant design solutions in low dimensions, they allow further reduction of the design dimension. Previous studies have noted that the adapted elliptic operators used to compute the eigenbases coincide with the gradient of the total-variation regularization functional [9], often used in image-processing and de-noising applications to identify and preserve sharp interfaces. The eigenfunctions of the adapted elliptic operators inherit these properties, as TO can be interpreted as the discovery and definition of optimal structural-void interfaces, and thus represent a particularly useful candidate basis to construct TO solutions.

The proposed method has several benefits that make it an attractive alternative to conventional density-based methods. We chiefly observe a significant reduction of the necessary design space dimension; in our 2D implementation, we reduce the design variable dimension by $1-2$ orders of magnitude with little loss in the structural performance. The truncated basis eliminates the need for filtering operations throughout the optimization procedure, thus avoiding the computational costs associated with filtering the solution and gradients each iteration. The adaptive scheme enables further reduction of the design dimension through multiple optimization passes and emphasizes refinement of the structural-void boundary. We note that restricting designs to any finite-dimensional subspace naturally constrains potential solutions; thus, by restricting designs to the eigenspace basis, we may not necessarily achieve designs with improved performance (i.e. lower objective function values) relative to designs using traditional element-wise parameterizations. While not explored here, our adaptive scheme could be paired with existing mesh adaptivity strategies, as has been explored in previous AEI studies [10], to refine the mesh and concentrate solution resolution near parameter discontinuities.

The rest of the paper is organized as follows. Section 2 introduces the formulation of the TO problems of interest. In Section 3, we formulate the EB design problem, presenting the associated eigenvalue problems used to construct the bases and the supplementary projection and regularization strategies. We then present in Section 4 the AEB strategy used to construct the sequence of eigenfunction bases; in particular, we demonstrate in Section 4.3 the 
accuracy and efficiency with which the adapted bases may represent a target structure, and provide criteria to select the basis dimension in Section 4.4. Finally, in Section 5, we demonstrate the merits of the approach in several examples of static displacement minimization design problems.

\section{2 | TOPOLOGY OPTIMIZATION PROBLEM}

\section{1 | Forward Problem}

We begin by introducing the generic topology optimization (TO) problem on which our reduced-basis strategy will be demonstrated, namely the design of linear-elastic structures with minimal displacement under static loading, subject to a volume fraction constraint. We represent the elastostatic boundary value problem governing the displacement response $\boldsymbol{u}$ of a body $\Omega$, under traction $\boldsymbol{\tau}$ and in the absence of body forces, in weak form: find $\boldsymbol{u} \in \mathcal{W}$ such that

$$
a(\boldsymbol{u}, \boldsymbol{w})=f(\boldsymbol{w}), \forall w \in \mathcal{W}
$$

where $\mathcal{W}:=\left\{\boldsymbol{w}: \boldsymbol{w} \in H^{1}(\Omega), \boldsymbol{w}=\mathbf{0}\right.$ on $\left.\Gamma_{D}\right\}$. The bilinear form a and linear functional $f$ are, as usual, defined by

$$
a(\boldsymbol{u}, \boldsymbol{w}):=\int_{\Omega} \epsilon[\boldsymbol{u}]: \mathbb{C}: \epsilon[\boldsymbol{w}] d \Omega, \quad f(\boldsymbol{w}):=\int_{\Gamma_{N}} \boldsymbol{w} \cdot \boldsymbol{\tau} d \Gamma_{N}
$$

where $\Gamma_{N}$ is the Neumann boundary, $\Gamma_{D}$ is the Dirichlet boundary, with $\Gamma_{N} \cup \Gamma_{D}=\partial \Omega, \Gamma_{N} \cap \Gamma_{D}=\varnothing, \epsilon[\boldsymbol{u}]=\frac{1}{2}\left(\nabla \boldsymbol{u}+\nabla \boldsymbol{u}^{T}\right)$ is the linearized strain tensor, and $\mathbb{C}=\mathbb{C}(\boldsymbol{x})(\boldsymbol{x} \in \Omega)$ is a positive, bounded, spatially-varying fourth-order constitutive tensor, where $\boldsymbol{x} \in \Omega$. We assume isotropic linear-elastic behavior, and thus may parameterize $\mathbb{C}(\boldsymbol{x})=\mathbb{C}(G(\boldsymbol{x}), \kappa(\boldsymbol{x}))$, with $G(\boldsymbol{x})$ and $\kappa(\boldsymbol{x})$ spatially-varying, real shear and bulk moduli. $\mathrm{n}$ the subsequent TO problems, $\Omega$ is a hold-all domain (called the "structural domain"), with eventual designs defined by spatial distribution of solid and void in $\Omega$.

We introduce a finite-dimensional approximation of $\boldsymbol{u}$ with a finite element discretization $\boldsymbol{u}_{h}(\boldsymbol{x})=[N(\boldsymbol{x})] \boldsymbol{d}$, with $[N]$ the matrix of global shape functions and $\boldsymbol{d} \in \mathbb{R}^{n_{d}}$ nodal displacement values. Substitution of this approximation for trial functions, and similarly for test functions, in (1) yields

$$
[K] \boldsymbol{d}=\boldsymbol{f}
$$

where $[K]$ is the stiffness matrix, $f$ is the forcing vector. We use the $[\cdot]$ notation to represent matrix quantities and boldface symbols for vector quantities. We use the following definitions for the construction of $[K]$ and $f$

$$
\begin{aligned}
{[K] } & :=\sum_{e} \int_{\Omega_{e}}[B]_{e}^{T}[D]_{e}[B]_{e} d \Omega_{e} \text { with }[D]_{e}:=G_{e}\left[D_{G}\right]+\kappa_{e}\left[D_{b}\right] \\
f & :=\sum_{e \in \Gamma_{N}} \int_{\Gamma_{N e}}[N]_{e}^{T} \tau d \Gamma_{e}
\end{aligned}
$$

in which $\sum_{e}$ represents the assembly operator over elements, $\Omega_{e}$ is the element domain, $[B]_{e}$ is the matrix of derivatives of element shape functions $[N]_{e},[D]_{e}$ is the elasticity matrix composed of shear and bulk components $\left[D_{G}\right]$, $\left[D_{\kappa}\right]$ with element-specific elastic constants $G_{e}$ and $\kappa_{e}$, and $\Gamma_{N e}$ is the portion of an element boundary on $\Gamma_{N}$. 


\section{2 | Structural Objective}

Structural performance is evaluated ijn terms of an objective $\mathcal{D}(\boldsymbol{d})$, a functional of displacements satisfying differentiability requirements consistent with the use of gradient-based methods. In this work, we select $\mathcal{D}(\boldsymbol{d})$ as a discrete approximation of the squared $L^{2}$-norm of displacements over a measured subdomain $\Omega_{m} \subseteq \Omega$

$$
\mathcal{D}(\boldsymbol{d})=\frac{1}{2 n_{e, m}} \boldsymbol{d}^{T}[Q] \boldsymbol{d}
$$

where $n_{e, m}$ is the number of elements in $\Omega_{m}$ and [Q] $\in \mathbb{R}^{n_{d} \times n_{d}}$ is a Boolean matrix, with ones in the diagonal entries corresponding to the targeted degrees of freedom and zeros elsewhere. We note that other common objectives, such as static compliance (e.g [12]) may be considered instead. The general goal of topology optimization (TO) is to find a structure, i.e. a spatial arrangement of given constitutive material, such that $\mathcal{D}(\boldsymbol{d})$ is minimized. The sought optimal structure usually may not exceed a certain volume, and can be subject to additional constraints.

\section{3 | Density Design Representation}

In a density-based TO formulation, a "fictitious density" field $\beta(\boldsymbol{x})$ describes the spatial distribution of the material within the domain, indicating distinct material phases or void and solid. This idea in particular underpins the Solid Isotropic Microstructure with Penalization (SIMP) model [12] adopted in this work, whereby elastic properties at any $\boldsymbol{x} \in \Omega$ interpolate the sets $\left\{\kappa_{0}, G_{0}\right\}$ and $\left\{\kappa_{1}, G_{1}\right\}$ of material parameters through

$$
\kappa(\boldsymbol{x})=\kappa_{0}+\Delta \kappa[h(\beta(\boldsymbol{x}))]^{p}, \quad G(\boldsymbol{x})^{p}=G_{0}+\Delta G[h(\beta(\boldsymbol{x}))]^{p},
$$

where $\Delta G:=\left(G_{1}-G_{0}\right), \Delta \kappa:=\left(\kappa_{1}-\kappa_{0}\right)$, the fictitious density $\beta$ takes values in [0,1], the function $h:[0,1] \rightarrow[0,1]$ is a smooth approximation of the Heaviside step function $[13,14]$ (see Sec. 3.1) and the penalization exponent $p$ (set in this work to $p=3$ ) serves to further discourage intermediate values of the "analysis density" field $h(\boldsymbol{\beta})$. Often, $\left\{\kappa_{0}, G_{0}\right\}$ is a very weak material approximating the void phase (while $\left\{\kappa_{1}, G_{1}\right\}$ defines the solid phase). Forward problems (1) are hence, in all cases, well-defined on the fixed structural domain $\Omega$.

Such density-based TO thus seeks optimal topologies defined by the field $\beta(\boldsymbol{x})$ through the above SIMP model. The latter is usually given an elementwise-constant approximation, the field $\beta$ being parameterized by $\boldsymbol{\beta} \in \mathbb{R}^{n_{e}}$ (with $n_{e}$ the number of elements); the discretized SIMP model thus has the form

$$
\kappa_{e}=\kappa_{0}+\Delta \kappa\left[h\left(\beta_{e}\right)\right]^{p}, \quad G_{e}=G_{0}+\Delta G\left[h\left(\beta_{e}\right)\right]^{p} \quad \text { on } \Omega_{e},
$$

Additional density-filtering operations [2] must in this case be performed upon $\boldsymbol{\beta}$ to ensure mesh-independence and 0-1 valued designs;

We may represent the system in (2) as an operator equation linking $\boldsymbol{\beta}$ and $\boldsymbol{d}$ :

$$
c(\boldsymbol{\beta}, \boldsymbol{d}):=[K(\boldsymbol{\beta})] \boldsymbol{d}-\boldsymbol{f}=\mathbf{0}
$$




\section{4 | Topology Optimization Problem}

In this work, we will use a reduced-space formulation for the topology optimization problem, where the state variable $\boldsymbol{d}$ is represented as as an implicit function of $\boldsymbol{\beta}$. Any $\boldsymbol{\beta}^{\star} \in[0,1]^{n_{e}}$ defines, through the (discretized) SIMP model, a valid elastic material distribution in $\Omega$. Together with the assumed boundary condition setting, this implies invertibility of the stiffness matrix $[K(\boldsymbol{\beta})]$, and the discretized PDE system in (2) admits a unique solution $\boldsymbol{d}^{\star}=\boldsymbol{d}\left(\boldsymbol{\beta}^{\star}\right)$, which satisfies (5). Thus, $\boldsymbol{d}$ may be represented as a implicit function $\boldsymbol{d}=\boldsymbol{d}(\boldsymbol{\beta})$, which by the implicit function theorem [15] is continuously differentiable in $\boldsymbol{\beta}$. This allows to define a reduced objective $\widetilde{\mathcal{D}}$, a function of only the design $\boldsymbol{\beta}$, by

$$
\widetilde{\mathcal{D}}(\boldsymbol{\beta}):=\mathcal{D}(\boldsymbol{d}(\boldsymbol{\beta}))
$$

and formulate the optimization problem in reduced form as:

$$
\boldsymbol{\beta}^{\star}=\underset{\boldsymbol{\beta} \in \mathbb{R}^{n_{e}}}{\arg \min } \widetilde{\mathcal{D}}(\boldsymbol{\beta}), \quad \text { subject to: } \quad 0 \leq \beta_{e} \leq 1, \quad V_{C}(\boldsymbol{\beta}):=\sum_{e} \frac{\left|\Omega_{e}\right|}{|\Omega|} h\left(\beta_{e}\right)-v_{\max } \leq 0
$$

The inequality $V_{C} \leq 0$ above represents the volume fraction constraint upon the analysis density field, with $|V|$ denoting the volume of a domain $V$ and $v_{\max }$ the prescribed maximum volume fraction.

\section{5 | Derivative Evaluation}

In this section, we use an adjoint-based formulation to derive the gradient of the objective with respect to the densities. Adjoint-based formulations to compute derivatives are widely used, due to their computational efficiency, and are introduced for instance in [15]. Recalling that $\boldsymbol{d}$ is continuously differentiable in $\boldsymbol{\beta}$, we express the directional derivative of $\widetilde{\mathcal{D}}$ with respect to $\boldsymbol{\beta}$, in direction $\boldsymbol{p} \in \mathbb{R}^{n_{e}}$, through application of the chain rule:

$$
\left\langle\widetilde{\mathcal{D}}_{\beta}^{\prime T}, \boldsymbol{p}\right\rangle=\mathcal{D}_{d}^{\prime T}\left[\boldsymbol{d}_{\beta}^{\prime}\right] \boldsymbol{p}
$$

where $\left\langle S_{\boldsymbol{\beta}}^{\prime}, \boldsymbol{p}\right\rangle:=\lim _{\eta \rightarrow 0}[S(\boldsymbol{\beta}+\eta \boldsymbol{p})-S(\boldsymbol{\beta})] / \eta$ denotes the Gâteaux derivative (at $\boldsymbol{\beta}$ and in direction $\boldsymbol{p}$ ) of a functional $S$, and can be represented in the present discretized setting by $\boldsymbol{S}_{\boldsymbol{\beta}}^{\prime} \in \mathbb{R}^{n_{e}}$ such that $\left\langle\boldsymbol{S}_{\boldsymbol{\beta}}^{\prime}, \boldsymbol{p}\right\rangle=\boldsymbol{S}_{\boldsymbol{\beta}}^{\prime T} \boldsymbol{p}$. To eliminate the solution sensitivity $\boldsymbol{d}_{\boldsymbol{\beta}}^{\prime}$ in (7), we next define the adjoint variable $\boldsymbol{v}:=\boldsymbol{v}(\boldsymbol{\beta}) \in \mathbb{R}^{n_{d}}$ which solves the adjoint problem

$$
\left[c_{d}^{\prime}(\boldsymbol{\beta}, \boldsymbol{d}(\boldsymbol{\beta}))\right]^{T} \boldsymbol{v}=-\mathcal{D}_{d}^{\prime}(\boldsymbol{d}(\boldsymbol{\beta}))
$$

which, recalling definitions (5) of $c(\boldsymbol{\beta}, \boldsymbol{d})$ and (3) of $\mathcal{D}(\boldsymbol{d})$, is equivalently expressed as

$$
[K(\boldsymbol{\beta})] \boldsymbol{v}=-[Q] \boldsymbol{d}(\boldsymbol{\beta})
$$

Additionally, taking the derivative of (5) with respect to $\boldsymbol{\beta}$ yields

$$
\left[c_{d}^{\prime}(\boldsymbol{\beta}, \boldsymbol{d}(\boldsymbol{\beta}))\right]\left[\boldsymbol{d}_{\boldsymbol{\beta}}^{\prime}\right]=-\left[c_{\boldsymbol{\beta}}^{\prime}(\boldsymbol{\beta}, \boldsymbol{d}(\boldsymbol{\beta}))\right]
$$


On left-multiplying (8) by $\left[d_{\beta}^{\prime}\right]^{T}$ and (10) by $\boldsymbol{v}^{T}$ and using the resulting equality of the right-hand sides in (7), we express the partial derivative $\widetilde{\mathcal{D}}_{\beta_{e}}^{\prime}=\widetilde{\mathcal{D}}_{\beta}^{\prime T} e_{e}$ as

$$
\widetilde{\mathcal{D}}_{\beta_{e}}^{\prime}=\boldsymbol{v}^{T}\left[c_{\beta}^{\prime}\right] e_{e}=\left(\boldsymbol{v}_{e}^{T}\left[K^{\prime}\right]_{e} \boldsymbol{d}_{e}\right) h^{\prime}\left(\beta_{e}\right)
$$

where $\left[K^{\prime}\right]_{e}$ is the derivative of the element stiffness matrix with respect to $h_{e}=h\left(\beta_{e}\right)$, and $\boldsymbol{d}_{e}, \boldsymbol{v}_{e}$ are the forward and adjoint variable nodal values on element $\Omega_{e}$. We evaluate

$$
\left[K^{\prime}\right]_{e}=p\left[h\left(\beta_{e}\right)\right]^{p-1} h^{\prime}\left(\beta_{e}\right) \int_{\Omega_{e}}[B]_{e}^{T}\left(\Delta K\left[D_{G}\right]+\Delta G\left[D_{K}\right]\right)[B]_{e} d \Omega_{e}
$$

\section{3 | EIGENSPACE BASIS DESIGN FORMULATION}

Conventional density-based TO methods formulate the design problem of binary placement of material using the density field $\beta(\boldsymbol{x})$ which, in most discretized settings, is approximated with piecewise-constant element values as presented in Section 2 . The design problem thus suffers from the high dimensionality of the discretized design variable $\boldsymbol{\beta}$, whose size equals the number of finite elements used. Such conventional parameterizations require regularization - through filtering or homogenization operations - to ensure existence of solutions, to impose minimum lengthscales, and to avoid mesh-dependence or checkerboarding numerical issues [2]. Fine discretizations may improve resolution of the structural topology, at the cost of increasing design dimension, but in a regularized setting should not change the qualitative form of the structural solution. Structural designs are ultimately characterized by multi-element, rather than element-to-element, features, a concept not leveraged by element-wise or nodal parameterizations.

Prompted by these considerations, we propose a new, reduced-basis approximation of the density field $\beta(\boldsymbol{x})$ to address issues of solution dimensionality and regularity. Beginning in a continuous setting, we seek $\beta(\boldsymbol{x})$ as a finite linear combination of basis functions $\psi_{j}(\boldsymbol{x})$, with coefficients $b_{j} \in \mathbb{R}$ :

$$
\beta(\boldsymbol{x}):=\sum_{j=1}^{\ell} b_{j} \psi_{j}(\boldsymbol{x}),
$$

where each $\psi_{j} \in H^{1}(\Omega)$ is an eigenfunction of an elliptic operator defined over $\Omega$ and $\ell$ is intended to be much smaller than $n_{e}$. Specifically, $\psi_{j}$ solves the eigenvalue problem (EVP) with homogeneous Neumann boundary conditions:

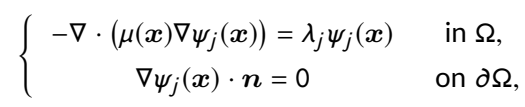

where $\mu(\boldsymbol{x})$ is a spatially-varying positive-definite tensor-valued coefficient $(\mu(\boldsymbol{x})=[I]$ corresponding to the usual Laplace operator) and $0=\lambda_{1} \leq \lambda_{2} \leq \ldots \leq \lambda_{\ell}$ are associated eigenvalues. We thus refer to representations of the form (11) as eigenspace basis (EB) expansions of $\beta$.

Note that we use Neumann eigenfunctions, rather than Dirichlet eigenfunctions as in previous EB-based parameter identification formulations $[9,10]$, because here the density at the boundary $\partial \Omega$ is not known beforehand.

We next set problem (12) in weak form, introducing a finite-dimensional approximation of the trial eigenfunctions $\psi_{j h}=\left[N_{1}\right] \psi_{j}$ and test functions, where $\left[N_{1}\right]$ are global scalar shape functions and $\psi_{j}$ the vector of eigenfunction nodal 
values, yielding the finite-dimensional generalized eigenvalue problem

$$
\left[K_{1}\right] \psi_{j}=\lambda_{j}\left[M_{1}\right] \psi_{j}
$$

$\left[K_{1}\right]$ and $\left[M_{1}\right]$ being the "stiffness" and "mass" matrices, respectively, defined by

$$
\left[K_{1}\right]:=\sum_{e} \int_{\Omega_{e}}\left[B_{1}\right]_{e}^{T}[\mu]_{e}\left[B_{1}\right]_{e} d \Omega_{e}, \quad\left[M_{1}\right]:=\sum_{e} \int_{\Omega_{e}}\left[N_{1}\right]_{e}^{T}\left[N_{1}\right]_{e} d \Omega_{e} .
$$

Here $\left[B_{1}\right]_{e}$ is the matrix of derivatives of the scalar element shape functions $\left[N_{1}\right]_{e}$, and $[\mu]_{e}$ is the tensor coefficient for the element. We obtain the element-wise $\beta_{e}(b)$ as a linear combination of nodal-valued eigenfunctions evaluated at element centroids $\overline{\boldsymbol{x}}_{e}$ :

$$
\beta_{e}(\boldsymbol{b})=\sum_{j=1}^{\ell} \psi_{j e} b_{j} \quad \text { with } \quad \psi_{j e}:=\left[N_{1}\left(\overline{\boldsymbol{x}}_{e}\right)\right]_{e} \psi_{j e}
$$

where $\psi_{j e}$ are the element nodal values for $\psi_{j}$ and $\psi_{j e}=\psi_{j}\left(\overline{\boldsymbol{x}}_{e}\right)$ is the element value of the discretized eigenfunction. We henceforth will use $\beta$ as the vector of $\beta_{e}$ and $[\Psi]=\left[\psi_{1}, \psi_{2}, \ldots, \psi_{\ell}\right]$ as the matrix of eigenfunctions.

As we will develop further in Section 4 , adaptive construction of the coefficient field $[\mu]_{e}$ will produce eigenbases that can efficiently represent piecewise-constant densities $\beta$ while avoiding costly additional filtering operations thanks to their moderate dimension $\ell$. Before that, we next describe additional operations performed upon the EB-expanded density to ensure convergence to binary material designs and finite values of coefficients $b_{j}$.

\subsection{Logistic transform}

In conventional density-based methods, regularization methods such as filtering operations ensure the existence and mesh-independence of solutions, but produce undesirable intermediate density values [2]. High-contrast solution quality is regained through "multiple-field strategies", where Heaviside function approximations $h$ are applied to filtered densities $\beta$ to produce the "analysis density" $h(\beta)$ that is finally input into the penalized material model (4) $[16,14]$. In our formulation, the truncated EB restricts the design space, thereby inducing density regularization as would a filter operation; this allows us to dispense with additional filtering operations. On the other hand, our adopted EB expansion of $\beta$ makes it difficult to enforce meaningful constraints on the density coefficients $b$. For this reason, we use a Heaviside-approximator $h$ that is a one-to-one $\mathbb{R} \rightarrow(0,1)$ function so as to ensure that analysis density values input into the SIMP model $(4)$ are in $(0,1)$. Accordingly, we adopt for $h$ an element-wise logistic function

$$
h\left(\beta_{e} ; \zeta\right):=\frac{1}{1+e^{-\zeta\left(\beta_{e}\right)}}
$$

parameterized by a constant $\zeta>0$. We note for later reference that

$$
h^{\prime}(\beta ; \zeta)=\frac{\zeta e^{-\zeta \beta}}{\left(1+e^{-\zeta \beta}\right)^{2}}
$$

which shows in particular that $\zeta$ is the slope of $h(\cdot ; \zeta)$ at $\beta=0$. Since $h(\cdot ; \zeta)$ converges to the Heaviside step function as $\zeta \rightarrow \infty$, increasing $\zeta$ allows to encourage high-contrast analysis density, with negative and positive values of $\beta_{e}$ yielding analysis densities $h\left(\beta_{e}\right)$ close to 0 and 1, respectively; see Fig. 1. 


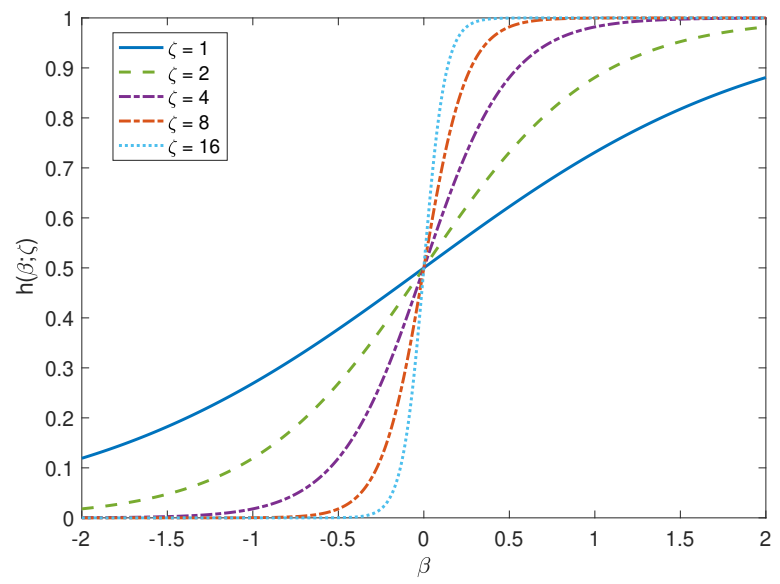

FIGURE 1 Logistic function $\beta \mapsto h(\beta ; \zeta)$, for various values of the slope parameter $\zeta$.

\section{2 | Double-Well Potential Regularization}

In minimum-displacement topology optimization, under material penalization and volume constraints, optimal solutions encourage placement of full material or complete elimination of material (i.e. $h\left(\beta_{e}\right)=0$ or $\left.h\left(\beta_{e}\right)=1\right)$ to maximize the structural stiffness for a given volume [2], which the logistic function $h$ achieves only in the limit $\beta_{e} \rightarrow \pm \infty$ for a finite $\zeta$. Bound constraints cannot be placed upon $\boldsymbol{b}$, as the ideal magnitude for coefficients is not known a priori. To control the magnitude of the $\beta$ field, we instead propose to add to the objective a double well potential (DWP) functional $W(\beta)$, defined in a continuous setting by

$$
W(\beta)=\frac{1}{|\Omega|} \int_{\Omega} w(\beta(\boldsymbol{x})) d \Omega, \quad \text { with } \quad w(\beta):=(\beta+1)^{2}(\beta-1)^{2}
$$

The DWP density $w$, a quartic polynomial plotted in Fig. 2 , is strictly positive, except at values $\beta= \pm 1$. The DWP regularization offers the attractive benefit of clustering $\beta$ near \pm 1 , penalizing densities in $(-1,1)$, thus providing a control on transitions between solid and void, as well those with large magnitude, preventing excessively large $b_{i}$ values. We can exploit the bimodal quality of $\boldsymbol{\beta}$ solutions regularized by the DWP term in adaptation and compression of the eigenfunction basis, as we will describe in Section 4. Our use of the DWP term for regularization of the basis coefficients is similar to the study in [17], which uses a DWP penalty term to relax 0-1 constraints in a phasefield topology optimization formulation. Interplay of the logistic function and the weighting applied to the DWP term controls the convergence of the solution to optimal topology with nearly binary analysis density values. Upon discretization and insertion of the element-wise EB expansion (14) into (16), the DWP becomes a function of $\boldsymbol{b}$ :

$$
W(\boldsymbol{b})=\frac{1}{|\Omega|} \sum_{e}\left|\Omega_{e}\right| w\left(\beta_{e}(\boldsymbol{b})\right)
$$




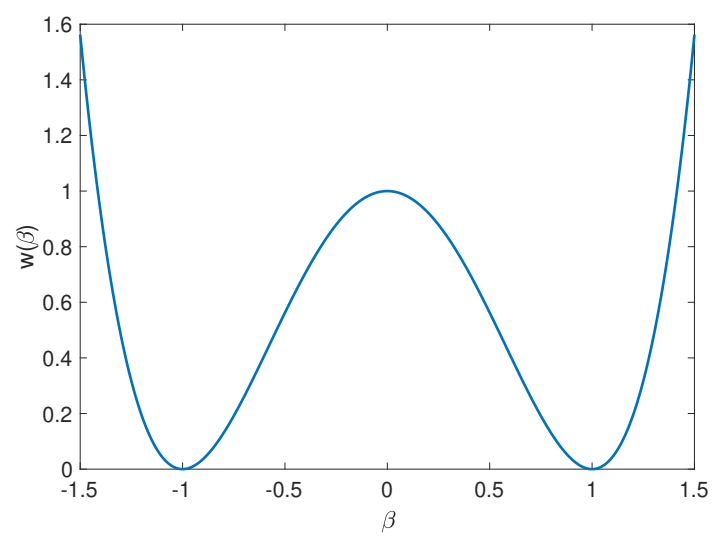

FIGURE 2 Double-well potential density $\beta \mapsto w(\beta)$.

\section{3 | Eigenspace Basis Formulation of Design Problem}

We may restate the topology optimization problem, now seeking the optimal basis coefficients $b \in \mathbb{R}^{\ell}$. Let the objective for the EB design problem, combining the structural performance and the DWP term, be

$$
\mathcal{J}(\boldsymbol{d}, \boldsymbol{b}):=\mathcal{D}(\boldsymbol{d})+\alpha W(\boldsymbol{b})
$$

where $\alpha$ is a scalar coefficient. We use similar arguments as in Section 2.4 to pose a reduced-space formulation. With the density now represented by the EB expansion (11), we recast (5) in the form $c(\boldsymbol{b}, \boldsymbol{d}):=c(\boldsymbol{\beta}(\boldsymbol{b}), \boldsymbol{d})=\mathbf{0}$. This system admits a unique solution $\boldsymbol{d}$ for any given $\boldsymbol{b} \in \mathbb{R}^{\ell}$, and $\boldsymbol{d}$ may be represented as a differentiable implicit function $\boldsymbol{d}=\boldsymbol{d}(\boldsymbol{b})$ using the arguments of Section 2.4. The reduced objective $\widetilde{J}(\boldsymbol{b})$ is accordingly defined as

$$
\widetilde{\mathcal{J}}(\boldsymbol{b}):=\mathcal{J}(\boldsymbol{d}(\boldsymbol{b}), \boldsymbol{b})=\mathcal{D}(\boldsymbol{d}(\boldsymbol{b}))+\alpha W(\boldsymbol{b}),
$$

and the topology optimization problem (6) becomes

$$
\boldsymbol{b}^{\star}=\underset{\boldsymbol{b} \in \mathbb{R}^{\ell}}{\arg \min } \tilde{\mathcal{T}}(\boldsymbol{b}), \quad \text { subject to: } \quad v_{C}(\boldsymbol{b}):=\sum_{e} \frac{\left|\Omega_{e}\right|}{|\Omega|} h\left(\beta_{e}(\boldsymbol{b})\right)-v_{\max } \leq 0 .
$$

\subsection{Derivative Evaluation}

We then borrow from the derivation in Section 2.5 to express the gradient of the reduced objective $\widetilde{\mathcal{J}}(\boldsymbol{b})$ with respect to $b$. The directional derivative of $\widetilde{\mathcal{J}}$ in direction $\boldsymbol{p} \in \mathbb{R}^{\ell}$ may again be expressed through application of the chain rule:

$$
\left\langle\widetilde{\mathcal{T}}_{b}^{\prime}, \boldsymbol{p}\right\rangle=\left(\mathcal{J}_{d}^{\top} \boldsymbol{d}_{b}^{\prime}+\mathcal{J}_{b}^{T}\right) p
$$

Through substitution of the adjoint solution $\boldsymbol{v}$ from (9) and using (10) as in Sec. 2.5, we find,

$$
\widetilde{\mathcal{T}}_{b}^{\prime}=\left[c_{b}^{\prime}\right]^{T} v+\mathcal{J}_{b}^{\prime}
$$


which allows to express the discrete evaluation of partial derivatives $\widetilde{\mathcal{T}}_{b_{j}}^{\prime}=\widetilde{\mathcal{T}}_{b}^{\prime T} \boldsymbol{e}_{j}$ with respect to individual coefficients $b_{j}$ as

$$
\widetilde{\mathcal{J}}_{b_{j}}^{\prime}=\boldsymbol{v}^{T} \boldsymbol{c}_{b_{j}}^{\prime}+\alpha W_{b_{j}}^{\prime}, \quad \text { where } \quad \boldsymbol{v}^{T} \boldsymbol{c}_{b_{j}}^{\prime}=\sum_{e}\left(\boldsymbol{v}_{e}^{T}\left[K_{e}^{\prime}\right] \boldsymbol{d}_{e}\right) h^{\prime}\left(\beta_{e}\right) \psi_{j e}, \quad W_{b_{j}}^{\prime}=\sum_{e} \frac{\left|\Omega_{e}\right|}{|\Omega|} 4 \beta_{e}\left(\beta_{e}^{2}-1\right) \psi_{j e}
$$

with the derivative of the logistic function given by (15) and where $\psi_{j e}$ is the element value of the discretized eigenfunction $\psi_{i}$, see (14). We also readily evaluate the gradient of the volume inequality constraint as

$$
V_{C, b_{j}}^{\prime}=\sum_{e} \frac{\left|\Omega_{e}\right|}{|\Omega|} h^{\prime}\left(\beta_{e}\right) \psi_{j e}
$$

\section{4 | ADAPTIVE EIGENSPACE BASIS CONSTRUCTION}

Reduction of the design space dimension may be enhanced, and acceleration of the overall optimization process achieved, through a multi-step basis-update strategy. In this section, we present a strategy for adapting the EB on which $\beta$ is expanded, modelled after that presented in [10] for medium imaging. Through multiple optimization passes, the EB is reconstructed using information from the current solution to capture global structural features in low-dimensional form and concentrate variation near shifting structural-void interfaces. The proposed basis adaptation will permit consistent reduction in the dimension of the design space throughout the optimization process, as few, specialized eigenfunctions may be used to express the structural form.

\section{1 | Adapted Eigenvalue Problem Construction}

The topology optimization problem is solved in several "passes". In each pass, a new EB expansion of $\beta$ is set up and the reduced optimization problem (18) is solved using that expansion. The EB for each pass is computed from an EVP of the form (12) whose tensor coefficient $[\mu]$ is constructed as in [10], using the solution from the previous pass so as to obtain eigenfunctions that increasingly emphasize regions where $\nabla \beta$ is large. We now describe each pass.

Pass 1. In the first pass, the EB is made of eigenfunctions of the Laplacian eigenvalue problem, setting the coefficient matrix in (12) as $\left[\mu^{(1)}\right]_{e}=[I]$, where $[I]$ is the identity matrix. We obtain $\left[\psi^{(1)}\right]$ from (13), and then solve the optimization problem (18) to determine $\boldsymbol{b}^{(1)}$, the density $\boldsymbol{\beta}^{(1)}$ as given by (14) and the analysis density $h\left(\boldsymbol{\beta}^{(1)}\right)$.

Pass 2. In the second pass, we modify the elliptic operator in (12) so that its eigenfunctions will exhibit localized features in regions with large spatial density gradients. We interpolate $\boldsymbol{\beta}^{(1)}$ onto the nodal basis, obtaining nodal values $\boldsymbol{\beta}_{N}^{(1)}$, and then define a field of element-wise isotropic coefficients as

$$
\left[\mu^{(2)}\right]_{e}:=\frac{1}{\max \left(\left|\nabla \beta_{e}^{(1)}\right|, \epsilon\right)}[I],
$$

having set $\nabla \beta_{e}^{(1)}=\left[B_{1}\right]_{e} \boldsymbol{\beta}_{N e}^{(1)}$ (where $\boldsymbol{\beta}_{N e}^{(1)}$ are the nodal values of $\boldsymbol{\beta}_{N}^{(1)}$ for element $\Omega_{e}$ ) and where $\epsilon=10^{-6}$ serves to prevent the denominator from vanishing.

Eigenfunctions of the EVP (13) with $\left[\mu^{(2)}\right]_{e}$, which is inversely proportional to the density gradient norm, will tend to exhibit high-frequency, localized spatial variation in regions with low $\mu$ values and low-frequency (near-constant) 
values in regions with higher $\mu$ values [8]. We solve the EVP (13) for $\left[\psi^{(2)}\right]$ and then solve the optimization problem (18) to determine $\boldsymbol{b}^{(2)}$, the density $\boldsymbol{\beta}^{(2)}$ and the analysis density $h\left(\boldsymbol{\beta}^{(2)}\right)$.

Pass 3. In the third pass, we increase the order of the gradient term in the denominator of the coefficient field, further emphasizing the density gradient, by setting

$$
\left[\mu^{(3)}\right]_{e}=\frac{1}{\max \left(\left|\nabla \boldsymbol{\beta}_{N e}^{(2)}\right|^{2}, \epsilon\right)}[I]
$$

with the gradient term evaluated as in (19). We solve the $\operatorname{EVP}(13)$ with $[\mu]=\left[\mu^{(3)}\right]$ for $\left[\Psi^{(3)}\right]$, determine the coefficient $\boldsymbol{b}^{(3)}$ by solving the optimization problem (18) and compute the associated fields $\boldsymbol{\beta}^{(3)}$ and $h\left(\boldsymbol{\beta}^{(3)}\right)$.

Pass 4. In this final optimization pass, we construct spatially-varying, anisotropic coefficients for the EVP, increasing the order of the gradient denominator term in the direction of the density gradient, normal to the structural-void interface. The rotation matrix $[P]_{e}$ defined on each element (for two-dimensional problems) by

$$
[P]_{e}:=\frac{1}{\left|\nabla \boldsymbol{\beta}_{N e}^{(3)}\right|^{2}}\left[\begin{array}{cc}
\frac{\partial \boldsymbol{\beta}_{N e}^{(3)}}{\partial x_{1}} & -\frac{\partial \boldsymbol{\beta}_{N e}^{(3)}}{\partial x_{2}} \\
\frac{\partial \boldsymbol{\beta}_{N e}^{(3)}}{\partial x_{2}} & \frac{\partial \boldsymbol{\beta}_{N e}^{(3)}}{\partial x_{1}}
\end{array}\right]
$$

transforms the Cartesian coordinate directions to directions that are colinear and orthogonal to $\nabla \beta$ (i.e. orthogonal and colinear, respectively, to level curves of $\beta$ ), as illustrated in Fig. 3. We then construct the coefficient field

$$
\left[\mu^{(4)}\right]_{e}:=\frac{1}{\max \left(\left|\nabla \boldsymbol{\beta}_{\mathrm{Ne}}^{(3)}\right|, \epsilon\right)}[P]_{e}[C]_{e}[P]_{e}^{-1}, \quad \text { with } \quad[C]_{e}:=\left[\begin{array}{cc}
\frac{1}{\max \left(\left|\nabla \boldsymbol{\beta}_{\mathrm{Ne}}^{(3)}\right|^{2}, \boldsymbol{\epsilon}\right)} & 0 \\
0 & 1
\end{array}\right]
$$

where $[C]$ is the anisotropic tensor emphasizing the direction of $\nabla \beta$ in the rotated coordinates. We solve the EVP (13) with $[\mu]=\left[\mu^{(4)}\right]$, which yields the final adapted $\mathrm{EB}\left[\psi^{(4)}\right]$, then determine the coefficient $\boldsymbol{b}^{(4)}$ by solving the optimization problem (18). This results in the final structural design, in terms of the fields $\boldsymbol{\beta}^{(4)}$ and $h\left(\boldsymbol{\beta}^{(4)}\right)$.

In summary, the coefficient fields introduced in passes 2 to 4 feature increasing powers of $|\nabla \beta|$ in the denominator, which causes eigenfunctions to become increasingly localized in transition regions near structural-void discontinuities.

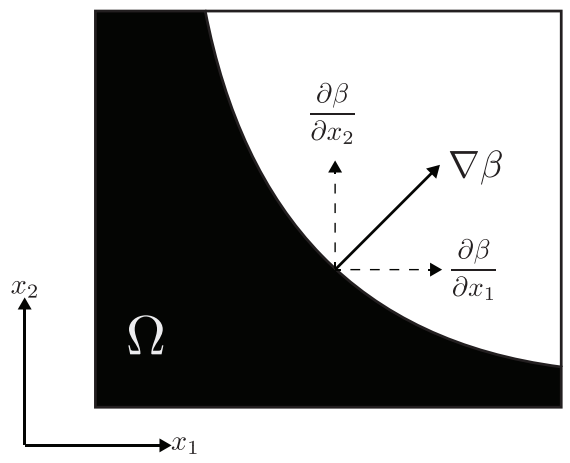

FIGURE 3 Rotation to coordinates aligned with density gradient. 


\section{2 | Justification for Adaptive Eigenfunction Decomposition}

The proposed adapted basis strategy has been shown to possess several characteristics that lend to its application in topology optimization. The sequence of Laplace eigenfunctions produced by Pass 1 , being a Hilbert basis of $L^{2}(\Omega)$, can be used to obtain Fourier series representations of arbitrary design patterns. However, due to the oscillatory nature of those eigenfunctions, Fourier series of piecewise-constant functions such as the density $\beta$ suffer from the Gibbs phenomenon and converge slowly. As a result, accurate Fourier series approximations of topologies with nuanced designs require large numbers of Laplace eigenfunctions. By contrast, the AEB functions produced by the complete, 4-pass construction of Sec. 4.1 are known to permit low-dimensional representation of arbitrary piecewise constant fields [11], as they have concentrated variation near transition regions between void and solid and near-constant values elsewhere. As noted by [8, 9], expanding $\beta$ upon the adapted eigenspace basis constitutes an operation analogous to total-variation regularization. Indeed, the smoothed total-variation operator $R_{T V}(\beta)$ is defined

$$
R_{T V}(\beta):=\frac{1}{2} \int_{\Omega} \sqrt{\left(|\nabla \beta|^{2}+\epsilon^{2}\right.} d \Omega
$$

where $\epsilon$ is a small constant. The second-order operators defined in the eigenvalue problems (12) (with coefficient fields constructed as in (19)) bear similarity to the gradient of $R_{T V}$ since for any direction of differentiation $p$ that vanishes on $\partial \Omega$ we have

$$
\left\langle\nabla R_{T V}(\beta), p\right\rangle=\int_{\Omega}\left(\frac{1}{\sqrt{|\nabla \beta|^{2}+\epsilon^{2}}} \nabla \beta\right) \cdot \nabla p d \Omega=\int_{\Omega}\left[-\nabla \cdot\left(\mu^{(2)}(\boldsymbol{x}) \nabla \beta\right)\right] p d \Omega
$$

Total-variation regularization penalizes high-frequency variation, rather than sharp discontinuities. The $T_{V}$ operator has been shown to preserve or capture sharp transitions in solution fields for image processing, de-noising, and parameter identification inverse-problem applications [18]. The proposed eigenfunctions behave similarly, able to capture contiguous constant regions of material and void while attenuating intra-region and high spatial frequency variation. The adapted eigenfunctions permit efficient representation of structural topologies, as density design parameterizations approximate sharp discontinuities between regions of void and full material. Increasing the power of the gradient norm in $\left[\mu^{(3)}\right]$, and further orienting anisotropic weights orthogonal to structural-void boundaries in $\left[\mu^{(4)}\right]$, enhances the edge-defining qualities of the eigenfunctions.

Importantly, our choice of including the DWP term in the objective functional $\widetilde{\mathcal{J}}(\boldsymbol{b})$ helps ensure that the developing density field exploits the edge-defining properties of the adapted eigenfunctions. By penalizing large and small values of $\boldsymbol{\beta}$, the DWP term helps cluster $\boldsymbol{\beta}$ in neighborhoods of the two minima $\boldsymbol{\beta}= \pm 1$ of the DWP. As a result, $\boldsymbol{\beta}$ is steered towards piecewise-constant values, ideally suited for low-dimensional representation by the adapted EB.

\section{3 | Adapted Basis Representation Accuracy}

Principal strengths of the AEB lie in its ability to conform to evolving piecewise constant density fields, and to represent such fields in low-dimensional form. In the following study, we demonstrate these qualities by computing projections of a target pattern $\boldsymbol{\beta}^{\star}$ upon adaptively-constructed bases of eigenfunctions, computed using Passes 1 to 4 as described in Sec. 4.1. The target design, shown in Figure 4, is a $2 \times 1 \mathrm{MBB}$ beam design containing $140 \times 70=9800$ elements; yellow-values have $\beta_{e}^{\star}=1$ and blue values, $\beta_{e}^{\star}=0$. We construct the bases $\left[\Psi_{\ell}^{(i)}\right]$ for each pass as stipulated in Sec. 4.1. We compute the first $\ell_{\max }=600$ eigenfunctions for each basis. In the first pass, we compute eigenfunctions of the Laplacian operator. In the subsequent passes $(i=2 \ldots 4)$, we compute adapted eigenbases, where the 




FIGURE 4 Target MBB design for representation study.

coefficient field $\left[\mu^{(i)}\right]_{e}$ entering the eigenvalue problem (13) for the $i$-th pass is constructed using $\boldsymbol{\beta}_{\ell_{\max }}^{(i-1)}$, the density field found by computing the $L^{2}$-projection of $\beta_{N}^{\star}$ upon the previous basis with dimension $\ell_{\max }$.

We compute the $L^{2}$-projections of $\beta_{N}^{\star}$ (the target densities in nodal projection form) onto the four bases [ $\Psi_{\ell}^{(i)}$ ] with increasing dimension $\ell$, by seeking for each $\ell \leq \ell_{\max }$ the $\ell$-vector $\boldsymbol{b}_{\ell}$ of coefficients solving the least-squares minimization problem

$$
\boldsymbol{b}_{\ell}^{\star}=\underset{b \in \mathbb{R}^{\ell}}{\arg \min } \frac{1}{2}\left\|\left[N_{1}\right] \boldsymbol{\beta}_{N}^{\star}-\left[N_{1}\right]\left[\Psi_{\ell}^{(i)}\right] \boldsymbol{b}_{\ell}\right\|_{L^{2}(\Omega)}^{2}
$$

whose solution $\boldsymbol{b}_{\ell}$ solves

$$
\left[M_{\ell}^{(i)}\right] b_{\ell}=\left[\Psi_{\ell}^{(i)}\right]^{T}\left[M_{1}\right] \boldsymbol{\beta}_{N}^{\star}
$$

where $\left[M_{\ell}\right]:=\left[\Psi_{\ell}^{(i)}\right]^{T}\left[M_{1}\right]\left[\Psi_{\ell}^{(i)}\right]$ is the projection of the mass matrix $\left[M_{1}\right]$ on $\operatorname{Span}\left(\left[\Psi_{\ell}^{(i)}\right]\right)$. We can then compute the relative $L^{2}$ residual $\epsilon_{\ell}^{(i)}$ between $\boldsymbol{\beta}_{N}^{\star}$ and $\boldsymbol{\beta}_{\ell}^{(i)}=\left[\Psi_{\ell}^{(i)}\right] \boldsymbol{b}_{\ell}$ as

$$
\epsilon_{\ell}^{(i)}:=\frac{\left\|\boldsymbol{\beta}_{N}^{\star}-\boldsymbol{\beta}_{\ell}^{(i)}\right\|_{M_{1}}}{\left\|\boldsymbol{\beta}_{N}^{\star}\right\|_{M_{1}}} .
$$

where we define the $L^{2}$-norm of the nodal discretized density field $\boldsymbol{\beta}_{N}$ as

$$
\left\|\boldsymbol{\beta}_{N}\right\|_{M_{1}}:=\sqrt{\boldsymbol{\beta}_{N}^{T}\left[M_{1}\right] \boldsymbol{\beta}_{N}}
$$

Results indicate the exceptional ability of the AEB to adapt to a given domain with discontinuous constant fields. Figure 5 shows resulting density fields produced from the $L^{2}$-projections of the target design upon the adapted eigenspace bases with dimensions $\ell=\{4,8,100,600\}$. The relative $L^{2}$ residuals $\epsilon_{\ell}^{(i)}$ are plotted in Fig. 6 against the basis dimensions $\ell$, demonstrating the improvement brought by each pass to the accuracy and efficiency with which the target density $\beta^{\star}$ can be projected onto the AEB. Projections using the initial Laplacian bases $\left[\Psi_{\ell}^{(1)}\right]$ slowly approximate $\beta^{\star}$; a few hundred eigenfunctions reasonably capture the structural features with $\epsilon_{\ell}^{(1)}$ reaching $14.57 \%$ for $\ell=\ell_{\max }=600$. However, projections using the subsequent bases achieve increasing accuracy: projection errors for $\ell=\ell_{\max }$ drop to $6.21 \%, 2.46 \%$ and $1.89 \%$, respectively, for passes 2,3 and 4 . The successive basis adaptation produces a basis specifically tailored to represent the piecewise constant target, and thus projection upon the AEB avoids the noisy error appearing in the projection upon the initial EB. The dimensional efficiency with which low errors are achieved also drops with each pass. Notably, the error precipitously decreases in Pass 4 for $\ell \geq 8$, with this accuracy clearly illustrated in Fig. 5 when comparing the Pass 4 projections for $\ell=4$ and $\ell=8$. Here, the basis dimension 
necessary to represent the target field nearly matches the number of distinct constant regions (i.e. 6) in the target.

Visual inspection of the EFs, as sampled in Fig. 7, illustrates the edge-defining capabilities of the adapted eigenfunctions. The Laplacian EFs $\psi_{j}^{(1)}$ from Pass 1 are oscillatory, as expected, with characteristic spatial frequency increasing with the index $j$. The second-pass EFs $\psi_{j}^{(2)}$ begin capturing the global structural shape at low indices $j$ while higher-index EFs emphasize localized density variations. After passes 3 and 4, the global structural patterns are captured with just the lowest-index EFs; for instance, the EF $\psi_{8}^{(4)}$ is seen to already represent specific, highly-localized regions of variation in the field $\beta$.

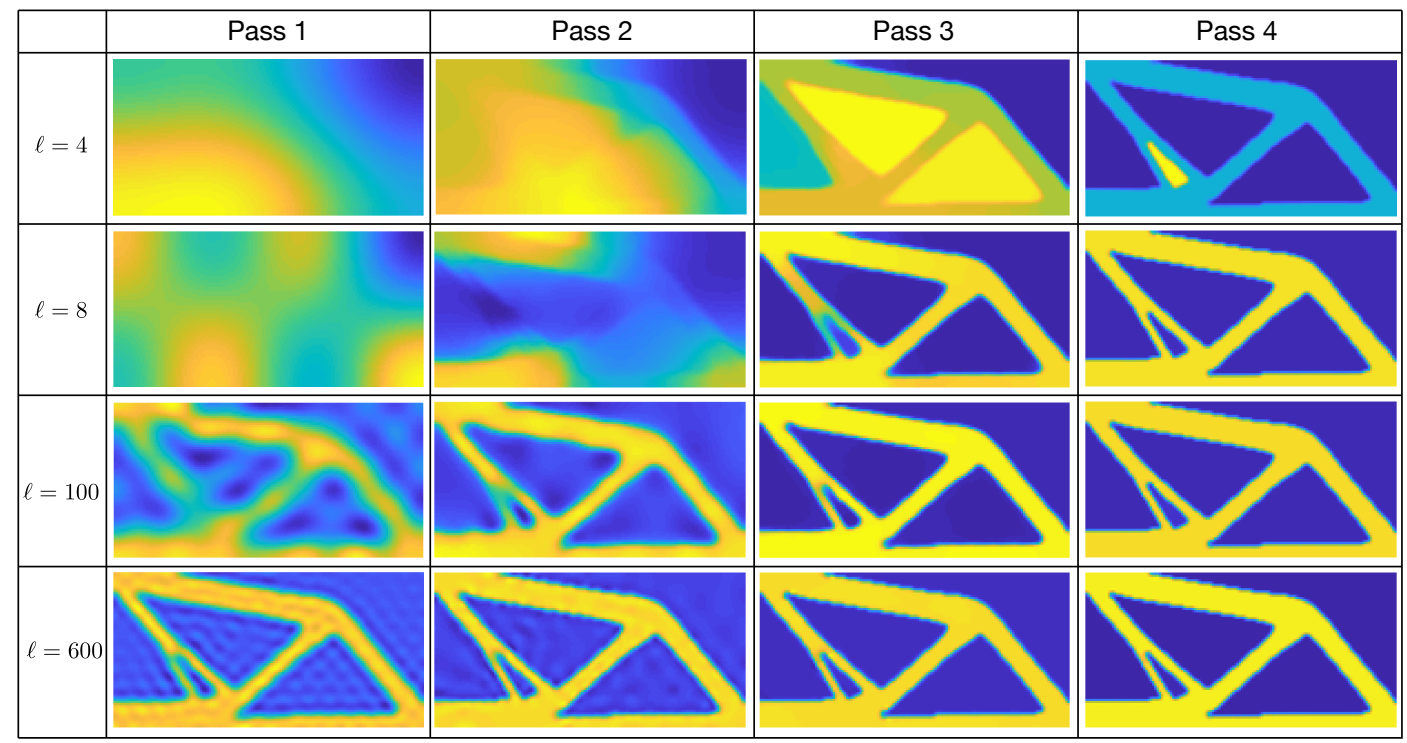

FIGURE $5 L^{2}$ projections of target density $\beta^{\star}$ onto eigenfunction bases of increasing dimension $\ell$.

\subsection{Criteria for Eigenspace Basis Selection}

The AEB design parameterization enables substantial reduction of the design space dimension $\ell$; selection of $\ell$, however, is a key factor in the quality of the structural solution. Basis truncation involves the inevitable trade-off between restriction of the search space and computational benefits afforded by reduced dimensionality.

Relatively ad-hoc criteria for basis dimension have been proposed for existing adaptive eigenspace bases in inverse problem settings. In frequency-domain inversion, the spatial resolution of the detected parameter features changes with measurement frequency; thus parameter inversion approaches have selected basis dimension with respect to the wavelength of measurement frequency [10] or have gradually increased basis dimension using frequencystepping algorithms [8]. However, we observe that geometric qualities of Laplacian eigenfunctions-namely the wavelength of spatial variation-may be inferred from their concurrently-calculated eigenvalues and used to convey length scale controls in TO design. Feature length scale and structural complexity are thus implicitly determined by the subset of eigenfunctions chosen, particularly during the first optimization pass. In the adaptive passes, basis selection can be further controlled to decrease dimension while ensuring minimal perturbation from the solution produced by one pass and the initial guess in the next pass. In this section, we will define criteria to select the initial and adapted 


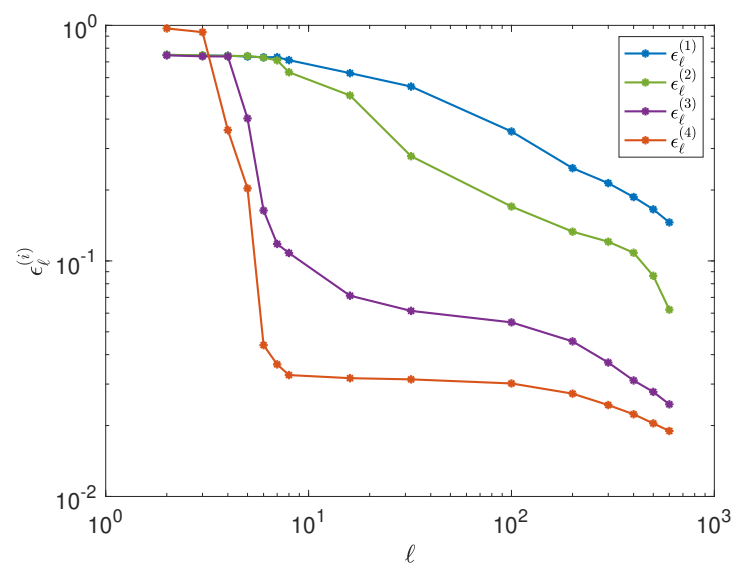

FIGURE 6 Relative $L^{2}$ residual $\epsilon_{\ell}^{(i)}$ (defined by (21)) between target and projected density fields, against EB dimension $\ell$.

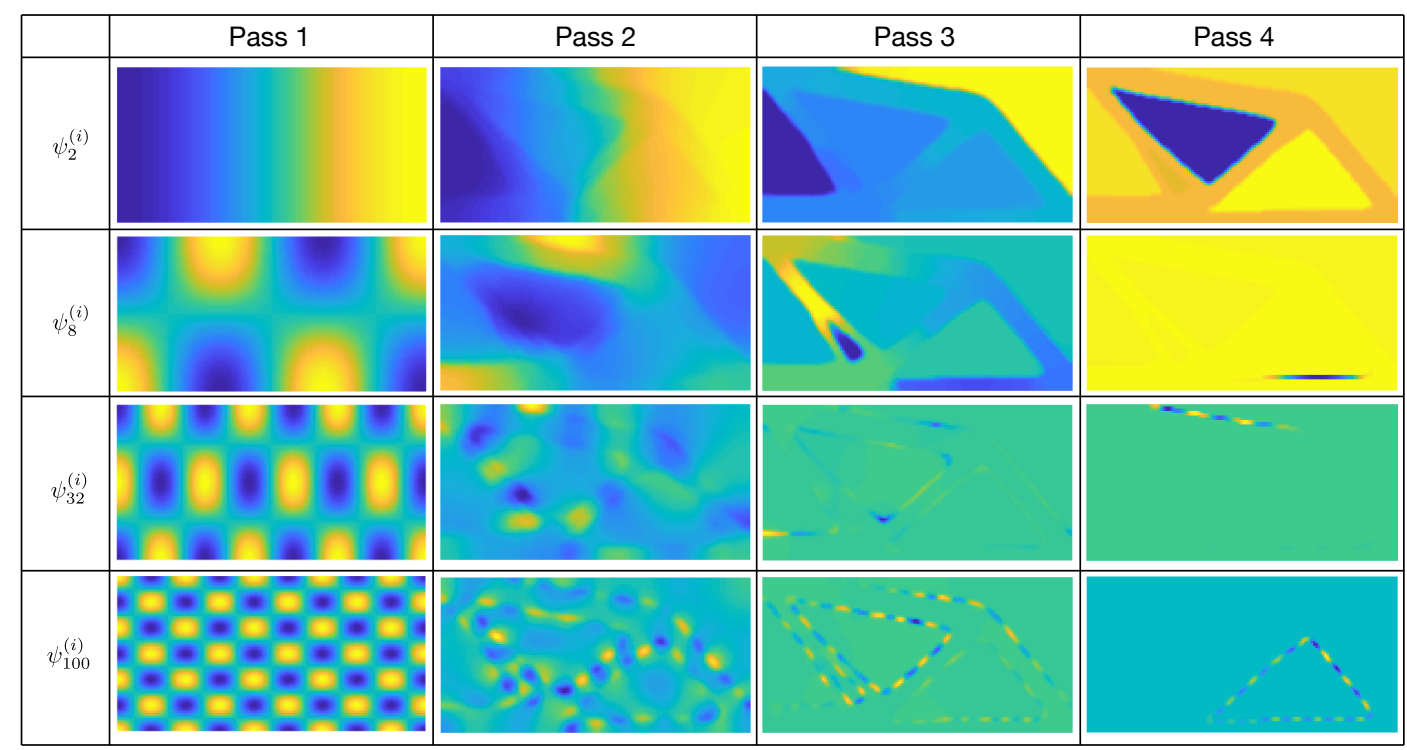

FIGURE 7 Example eigenfunctions $\psi_{j}^{(i)}$ computed from the eigenvalue problems for Passes 1 to 4.

eigenbasis dimensions.

\subsection{1 | Initial Pass}

We propose selecting the dimension $\ell$ of the basis $\left[\boldsymbol{\Psi}_{\ell}^{(1)}\right]$ by means of the characteristic spatial frequency of the Laplacian eigenfunctions. Indeed, expressing the eigenvalue $\lambda_{i}$ associated with a eigenfunction $\psi_{i}$ as a Rayleigh quotient, 
the characteristic wavelength $w_{i}$ of $\psi_{i}$ is

$$
w_{i}=2 \pi \sqrt{\frac{1}{\lambda_{i}}}, \quad \text { where } \quad \lambda_{i}=\frac{\int_{\Omega}\left|\nabla \psi_{i}\right|^{2} d \Omega}{\int_{\Omega} \psi_{i}^{2} d \Omega}
$$

Eigenfunctions associated with large eigenvalues $\lambda_{i}$ are characterized by small $w_{i}$, i.e. high-frequency spatial oscillation; truncation of the EB to eigenfunctions with lowest eigenvalues limits this short-wavelength spatial variation and implicitly controls the minimum length scale in $\beta$. Moreover, the maximum length scale of structural members can also be controlled through additional removal of low-frequency eigenfunctions, limiting large-wavelength variation in $\beta$. We thus evaluate $w_{i}$ for all computed eigenfunctions, cutting off eigenfunctions from either end of the spectrum and retaining in the eigenspace basis only those for which $w_{\min } \leq w_{j} \leq w_{\max }$. Wavelength thresholds $w_{\min }, w_{\max }$ may be selected to correspond approximately to the minimum (or maximum) feature width $d$ desired in the structural design, e.g by setting $w_{\min } \approx 2 d_{\text {min }}$.

We may tie this basis truncation to the effect of PDE-based filters. Existing PDE-based filter operations (e.g. [3]) compute a filtered version of $\beta$ by solving a heat-type equation with $\beta$ acting as "forcing" inputs, which essentially attenuates high-frequency content from $\beta$ in order to ensure solution existence. Lazarov et. al showed that PDE-based filters may be constructed for both low-pass and band-pass scenarios, enabling implicit control of small (and large) length scales appearing in structural solutions [19]. We can view the truncation of the basis $\left[\boldsymbol{\Psi}_{\ell}^{(1)}\right]$ as a straightforward filtering operation which explicitly removes frequency content from the solution spectrum, with the added benefits of being performed only once in the optimization problem and trivial applicability to domains of arbitrary shape.

We note that truncation with respect to a specified wavelength/frequency may not explicitly remove all frequency content outside specified frequency thresholds-as eigenfunctions may contain a combination of different spectral components centered at a mean frequency. This extraneous frequency content tends to have low spectral power, however, contributing little to the spatial characteristics of a given eigenfunction.

We can briefly demonstrate eigenfunction spectral qualities by computing the spatial Fast Fourier Transform (SFFT) of eigenfunctions and plotting their amplitude spectra. Eigenfunctions of a sample rectangular domain, and their respective SFFT, are shown in Fig. 8 . We add circles with radii equal to the dominant frequency predicted by the corresponding eigenvalue and see coincidence of the predicted dominant frequencies with the radial distribution of spectral power. Low-index eigenfunctions contain concentrated spectral content exactly at the predicted frequency, while high-index eigenfunctions have more-distributed frequency content, nevertheless concentrated radially at the predicted frequency. Thus, comparing these principal wavelengths to a threshold length scale $w_{\min } \leq w_{j} \leq w_{\max }$ can provide criteria to restrain the length scales represented within an eigenbasis.

\subsection{2 | Subsequent Adaptive Passes}

In selecting the adapted basis members in subsequent passes, we have several objectives: 1) to ensure that our initial guess on the new eigenfunction basis be close to our previous solution; 2) to utilize eigenfunctions that contribute most to the current design; and 3) to maintain or decrease the dimension of our basis with each successive pass. High-index eigenfunctions are typically characterized by highly-localized, sparse spatial variation that may contribute little towards representing, or improving, the solution. Accordingly, basis truncation is still be used to restrain high-frequency spatial variation. Continued compression of the basis is also desirable as it may enable the use of advantageous computational (e.g. second-order) methods. Taking these objectives in mind, we propose an $L^{2}$-error criterion to select the AEB dimension $\ell$ of the $\mathrm{EB}\left[\Psi_{\ell}^{(i)}\right]$ created in pass $i(i=2,3,4)$. 


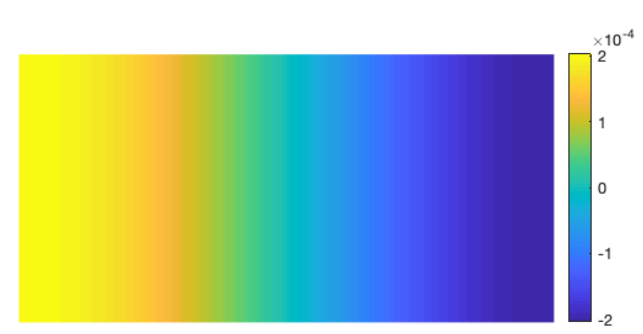

(a) Eigenfunction $\psi_{2}^{(1)}$

fig_fftphi2

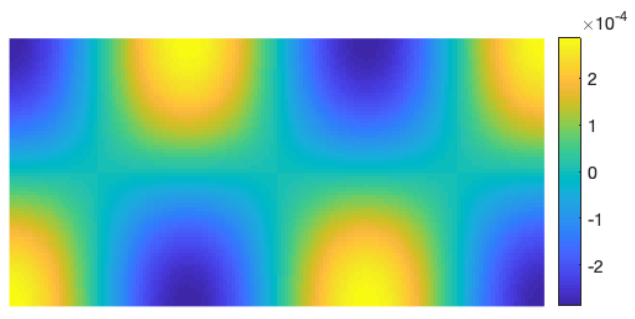

(c) Eigenfunction $\psi_{8}^{(1)}$

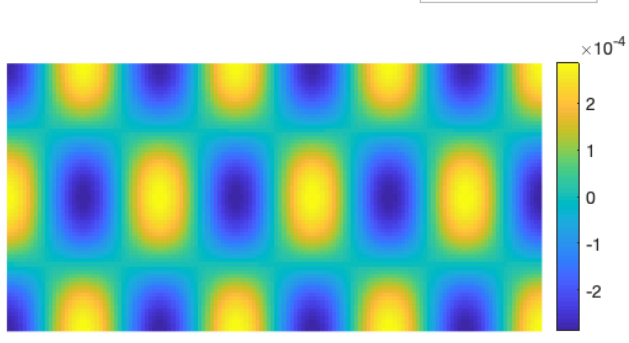

(e) Eigenfunction $\psi_{32}^{(1)}$

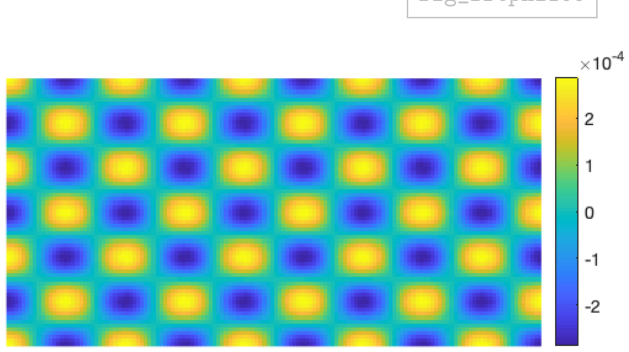

(g) Eigenfunction $\psi_{100}^{(1)}$

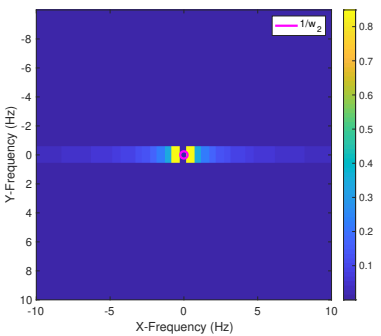

(b) Spatial FT of $\psi_{2}^{(1)}$

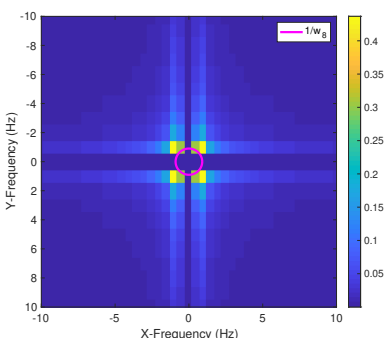

(d) Spatial FT of $\psi_{8}^{(1)}$

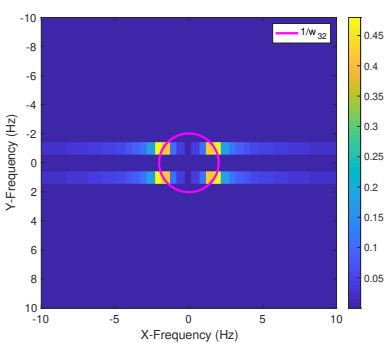

(f) Spatial FT of $\psi_{32}^{(1)}$



(h) Spatial FT of $\psi_{100}^{(1)}$

FIGURE 8 Laplacian eigenfunctions $\psi_{j}^{(1)}$ of a $2 L \times L$ rectangular MBB domain, and corresponding amplitude spectra of SFFTs in $(x, y)$ frequency space. Pink circles indicate dominant frequencies predicted from eigenvalues.

Let $\boldsymbol{\beta}^{(i-1)}$ be the density solution produced by pass $i-1$, and let $\left[\Psi_{j}^{(i)}\right]$ denote the adapted basis truncated to the first $j$ eigenfunctions, ordered by increasing eigenvalues. We first evaluate the following relative $L^{2}$-residuals between 
$\boldsymbol{\beta}^{(i-1)}$ and its projections onto expanding subsets of the new basis:

$$
\epsilon_{0, j}^{(i)}:=\frac{\left\|\boldsymbol{\beta}^{(i-1)}-\left[\Psi_{j}^{(i)}\right] \boldsymbol{b}_{0, j}^{(i)}\right\|_{M_{1}}}{\left\|\boldsymbol{\beta}^{(i-1)}\right\|_{M_{1}}} .
$$

where $\boldsymbol{b}_{0, j}^{(i)} \in \mathbb{R}^{j}$ is the vector of the coefficients for the $L^{2}$ projection of $\boldsymbol{\beta}^{(i-1)}$ upon $\left[\boldsymbol{\Psi}_{j}^{(i)}\right]$, as computed with (20). Making use of the $\left[M_{1}\right]$-orthogonality of the $\psi_{j}$, we note that the $L^{2}$-projections of $\beta^{(i-1)}$ upon single eigenfunctions $\psi_{k}^{(i)}$ are all mutually $\left[M_{1}\right]$-orthogonal. Lower-index eigenfunctions are generally, but not necessarily, associated with larger $L^{2}$-projection components. Thus, the eigenfunction basis can easily be reordered by relative decrease in $L^{2}$-projection residual achieved by each new eigenfunction, to prioritize eigenfunctions that contribute most to the current design solution. The decrease of relative $L^{2}$ residual resulting from adding eigenfunction $\phi_{j}$ to the EB is

$$
\hat{\epsilon}_{0, j}^{(i)}:=\epsilon_{0, j-1}^{(i)}-\epsilon_{0, j}^{(i)}
$$

We may then reorder the eigenfunctions $\psi_{j}^{(i)}$ according to decreasing $\hat{\epsilon}_{0, j}^{(i)}$. Figure 9 , which shows $\hat{\epsilon}_{0, j}^{(i)}$ versus index $j$ for the eigenvalue-ordered and error-ordered eigenfunctions computed in the representation study of Section 4.3, demonstrates the added benefit of the latter reordering method. We finally define the relative decrease of relative projection error due to a given eigenfunction as

$$
c_{j}^{(i)}=\frac{\hat{\epsilon}_{0, j}^{(i)}}{\hat{\epsilon}_{0,1}^{(i)}+\ldots+\hat{\epsilon}_{0, j-1}^{(i)}},
$$

which, by construction, decreases monotonically with $j$. We may then truncate the reordered basis $\left[\Psi_{\ell}^{(i)}\right]$ on the basis of a tolerance on $c_{j}^{(i)}$, indicating that the $L^{2}$ error is now negligibly decreasing if basis dimension is further increased.

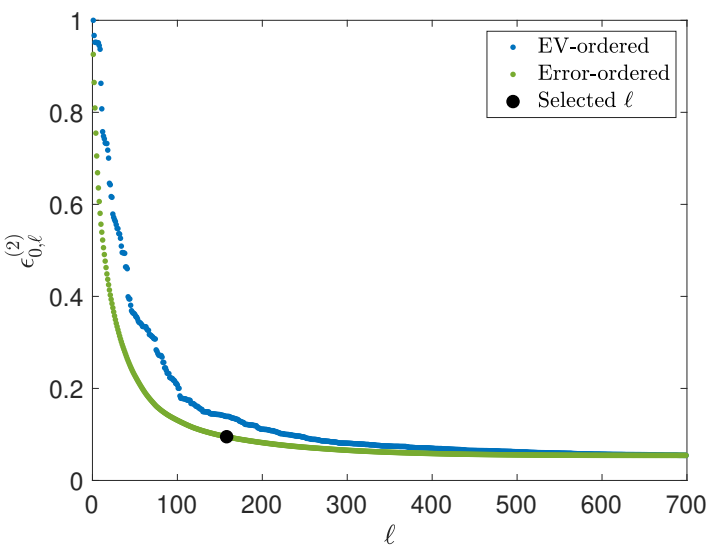

FIGURE 9 Relative $L^{2}$ residuals $\epsilon_{0, \ell}^{(i)}$ between density solution $\boldsymbol{\beta}^{(i-1)}$ from previous pass and its projection upon the Pass- $i$ EB, with increasing EB dimension $\ell$ (here, $i=2$ ). Reordering of eigenfunctions along relative residual decreases $\hat{\epsilon}_{0, j}^{(i)}$, rather than eigenvalues $\lambda_{j}$, prioritizes selection of basis functions with largest contributions to current solution. 

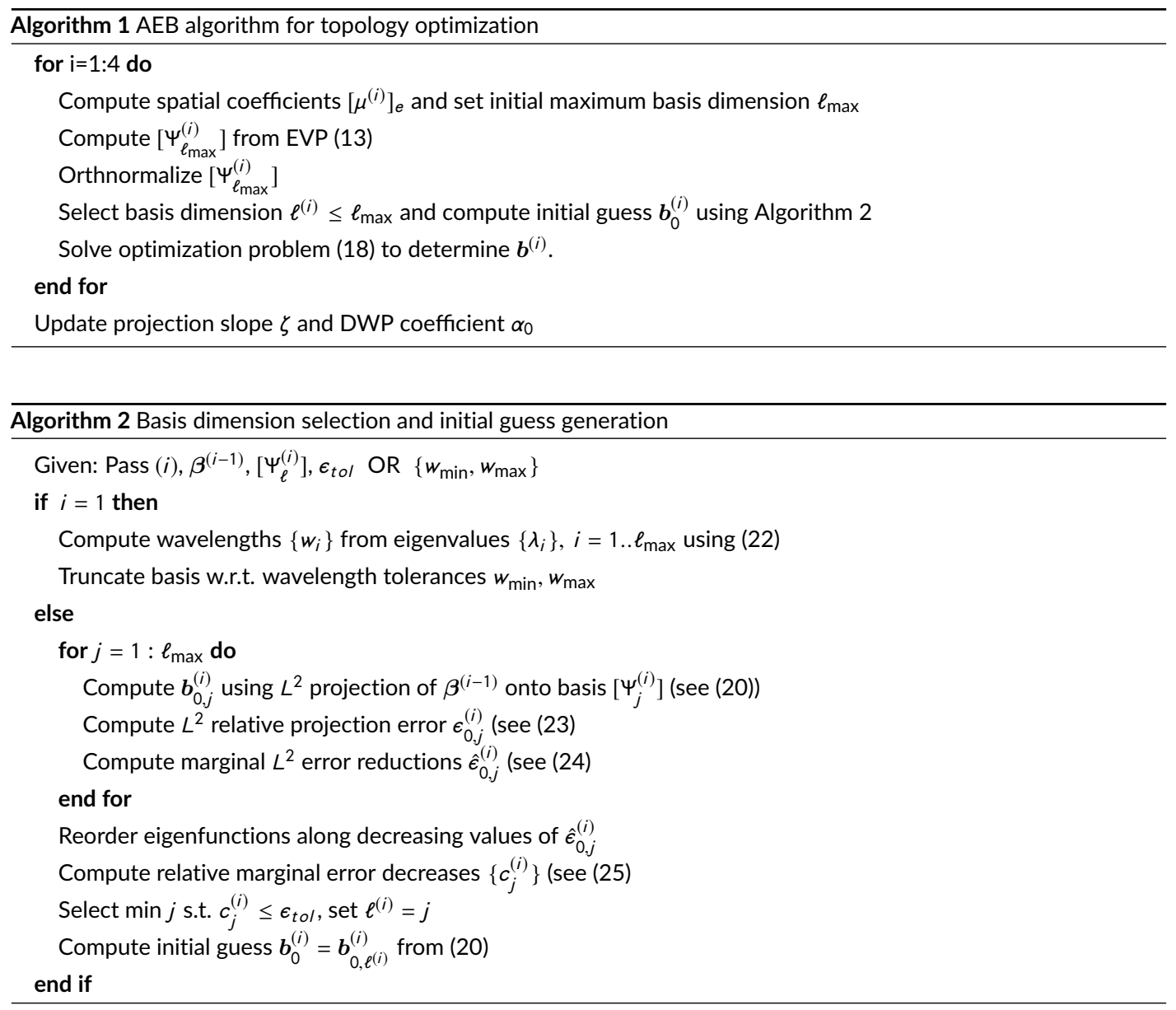

We summarize the AEB algorithm for solving the topology optimization problem in Algorithm 1 and the procedure for basis dimension selection in Algorithm 2.

\section{5 | DESIGN STUDIES}

In this section, we demonstrate the performance of the AEB method in solving topology optimization problems. We examine the effects of basis dimension upon solution quality and the solution improvement gained through basis adaptation. We benchmark our results against results from conventional density-based design methods (CDM), examining structural performance comparisons and potential computational cost savings. We furthermore provide guidance on implementation of the parameter continuation schemes that enable consistent convergence of the AEB method to 0-1 solutions. We discuss implementation details in Appendix A.

Except in Sec. 5.2, we will consider two prototypical design cases for linear-elastic topology optimization. The first one is the "MBB beam" (Fig. 10a), which consists of a simply-supported beam subjected to a midspan loading. Using symmetry, only half of the structural domain is modeled. The goal is to minimize the downward displacement at the 
midspan node (indicated in blue in Fig. 10a). The second one is a cantilever beam (Fig. 10b), subject to fixed-support at its left edge and a downward traction along a portion $\Gamma_{m}$ of the right edge. The goal in this case is to minimize displacements on $\Gamma_{m}$ (shown in blue in Fig. 10b). All examples in this section use a two-phase interpolation of elastic properties of the form (4), with void phase properties $\left\{G_{0}, \kappa_{0}\right\}=\left\{1 \times 10^{-5}, 4 \times 10^{-5}\right\}$ and solid phase $\left\{G_{1}, \kappa_{1}\right\}=\{1,4\}$.

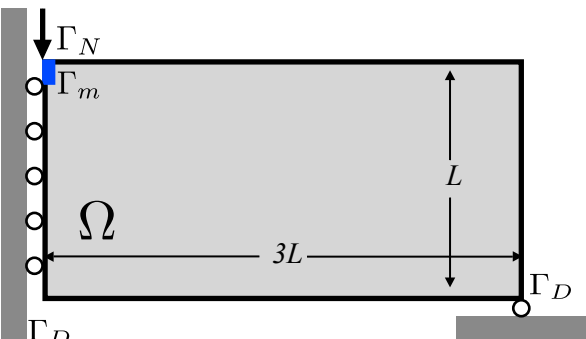

(a) MBB beam design domain

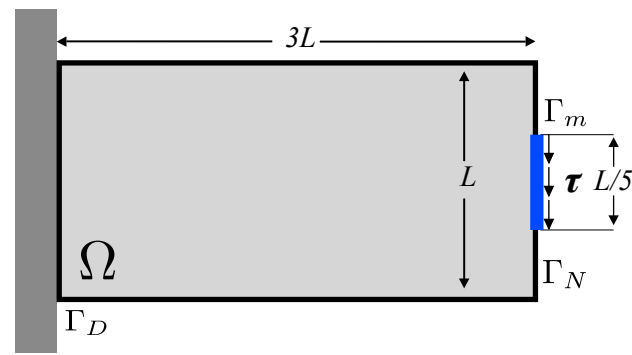

(b) Cantilever beam design domain.

FIGURE 10 Linear elastic design cases: notation, geometry of structural domain $\Omega$. Void and solid phases respectively defined by $\left\{G_{0}, \kappa_{0}\right\}=\left\{1 \times 10^{-5}, 4 \times 10^{-5}\right\}$ and $\left\{G_{1}, \kappa_{1}\right\}=\{1,4\}$.

The AEB strategy applied to these examples is implemented in a two-dimensional FE MATLAB code and solved with the MATLAB fmincon interior-point method [20]. For comparison purposes, a CDM is also implemented to benchmark solution quality, though using the MATLAB globally-convergent Method of Moving Asymptotes algorithm to solve the optimization problem [21]. We evaluate structural performance of the designs, as measured by their structural objective $\mathcal{D}(\boldsymbol{d})$ and gray fraction $f_{g}(\boldsymbol{\beta})$. The latter, computed as

$$
f_{g}(\boldsymbol{\beta}):=\frac{4}{|\Omega|} \sum_{e}\left|\Omega_{e}\right| h\left(\beta_{e}\right)\left(1-h\left(\beta_{e}\right)\right) .
$$

was introduced in [22] and measures the residual fraction of gray material in the optimal analysis density field $h(\boldsymbol{\beta})$. We also study the convergence behavior and the effects of various algorithmic parameters on the solution.

\section{1 | Study: Basis Dimension}

We first evaluate the effect of initial EB dimension $\ell^{(1)}$ upon design quality with a series of designs (trials 1 to 6 ) which differ by varying $\ell^{(1)}$ through the choice of wavelength thresholds $w_{\min }, w_{\max }$. We consider the cantilever beam case (Fig. 10b), using $180 \times 60$ bilinear, four-node elements under plane-stress conditions and subject to a $50 \%$ volume fraction constraint (i.e. $v_{\max }=0.5$ in problem (18)). Void and solid phases are as previously defined (see also Fig. 10).

We use only the Laplacian eigenfunctions $\psi_{j}^{(1)}$ (pass 1, $[\mu]_{e}=[I]$ ) for our design basis, and evaluate the characteristic wavelength $w_{j}$ for each $\psi_{j}^{(1)}$ using (22). For trials 1 to 4 , we truncate the design subspace based upon a decreasing minimum wavelength $w_{\min }$, inducing increasing dimensions $\ell^{(1)}$ of the $\mathrm{EB}\left[\Psi_{\ell}^{(1)}\right]$. For trials 5 and 6 , we instead perform "band-pass" truncation, eliminating also low-frequency eigenfunctions by way of an upper wavelength limit $w_{\text {max }}$. We use continuation schemes for the slope $\zeta$ and the DWP coefficient $\alpha$ in the objective $\widetilde{\mathcal{J}}(\boldsymbol{b})$ (see (17)). We initialize $\zeta=2$ and apply an update factor of 1.5 every 100 iterations, or upon reaching a local minimum, while we initialize $\alpha_{0}=10^{-4}$ and apply an update factor of 1.2 each time $\zeta$ is updated. Each example is limited to at most 750 total 
iterations. In the interior point method, multiple function evaluations may be necessary within a iteration, so we also report function evaluation count to reflect true computational cost. Design statistics, including final displacement objective and gray fraction achieved by the outcome $\boldsymbol{\beta}^{(1)}=\boldsymbol{\beta}\left(\boldsymbol{b}^{(1)}\right)$, are reported in Table 1. Figure 12 shows the obtained designs.

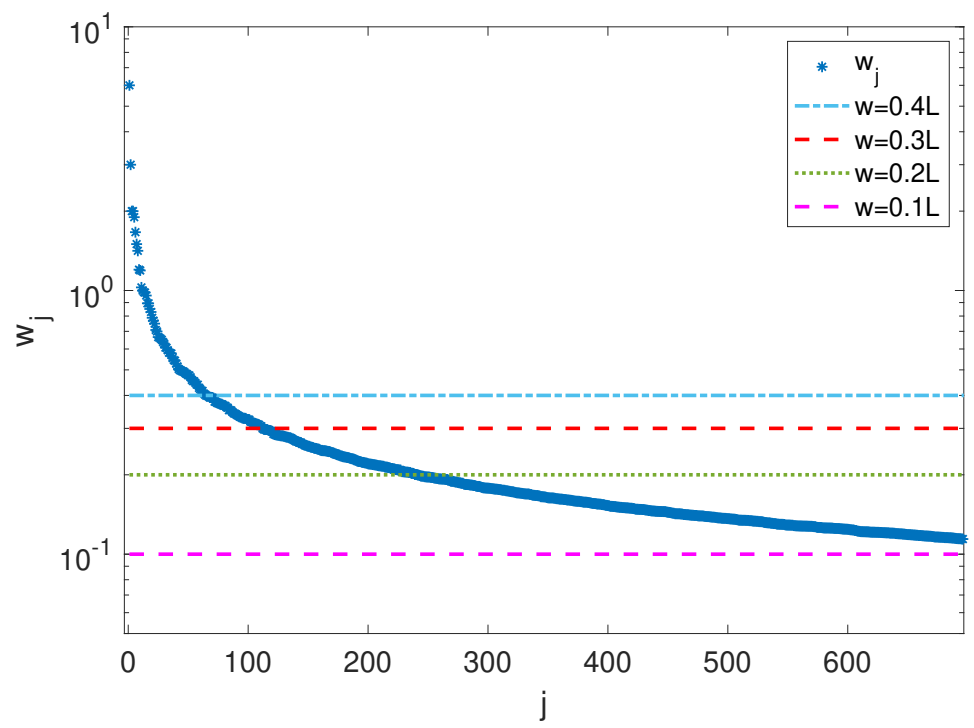

FIGURE 11 Characteristic wavelengths $w_{j}$ of the eigenfunctions $\psi_{j}^{(1)}$ (ordered along increasing eigenvalues $\lambda_{j}$ ), as defined by (22). Dashed lines show the cutoff thresholds used in design studies.

\begin{tabular}{|c|c|c|c|c|c|c|c|}
\hline Trial & $\left\{w_{\min }, w_{\max }\right\}$ & $\ell^{(1)}(\mathrm{EB}$ dim. $)$ & $\mathcal{D}\left(\boldsymbol{d}\left(\boldsymbol{b}^{(1)}\right)\right)$ & $f_{g}\left(\boldsymbol{b}^{(1)}\right)$ & $W\left(\boldsymbol{b}^{(1)}\right)$ & Iter Count & Func Count \\
\hline \hline 1 & $\{0.4 L,-\}$ & 63 & $9.25710^{1}$ & 0.0778 & $1.01810^{1}$ & 703 & 742 \\
\hline 2 & $\{0.3 L,-\}$ & 110 & $8.36010^{1}$ & 0.0902 & 9.071 & 629 & 669 \\
\hline 3 & $\{0.2 L,-\}$ & 239 & $7.60410^{1}$ & 0.0520 & 2.156 & 597 & 630 \\
\hline 4 & $\{0.1 L,-\}$ & 699 & $7.33610^{1}$ & 0.0240 & 1.098 & 439 & 490 \\
\hline 5 & $\{0.1 L, 0.4 L\}$ & 636 & $1.17310^{2}$ & 0.0324 & $3.10510^{1}$ & 658 & 750 \\
\hline 6 & $\{0.1 L, 0.2 L\}$ & 459 & $2.03410^{2}$ & 0.0297 & $1.09210^{2}$ & 620 & 669 \\
\hline
\end{tabular}

TAB LE 1 Eigenspace basis design (Pass 1): effects of EB dimension $\ell^{(1)}$ upon structural performance $\mathcal{D}(\boldsymbol{d})$. Wavelength limits $w_{\min }, w_{\max }$ are set relative to the domain height $L$.

The EB method successfully obtains acceptable cantilever designs using EB dimensions $\ell^{(1)}$ nearly two orders of magnitude smaller than a conventional, element-wise density parameterization. We observe that the lower-dimensional EB solutions (e.g. Trial 1) yield large displacement objective values $\mathcal{D}(\boldsymbol{d})$, while solutions obtained with expanded EB subspaces (e.g. Trial 4) are able to achieve lower values of $\mathcal{D}(\boldsymbol{d})$. We do observe structural performance improve- 


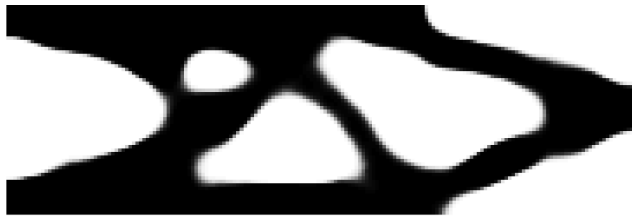

(a) Trial 1: $w_{\min }=0.4 L$

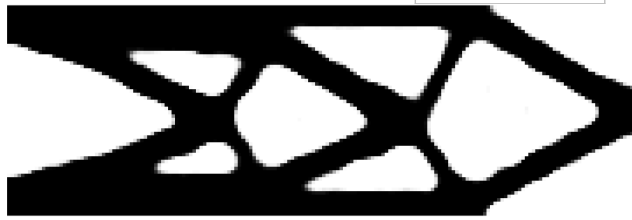

(c) Trial 3: $w_{\min }=0.2 L$

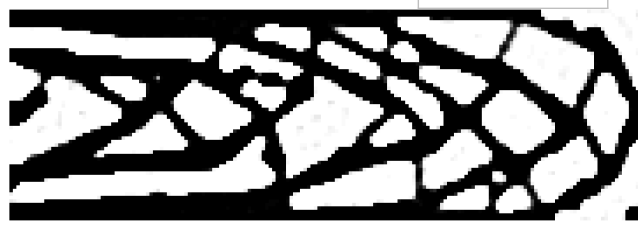

(e) Trial 5: $\left\{w_{\min }, w_{\max }\right\}=\{0.4 L, 0.1 L$

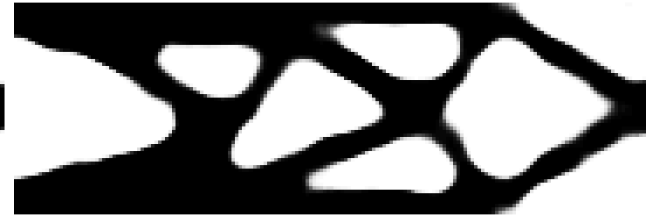

(b) Trial 2: $w_{\min }=0.3 \mathrm{~L}$

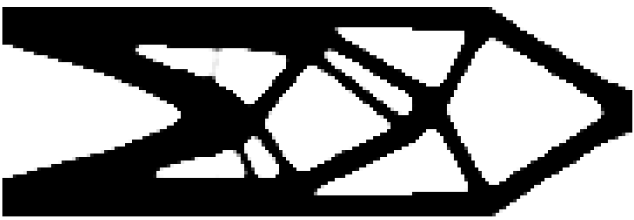

(d) Trial 4: $w_{\min }=0.1 \mathrm{~L}$

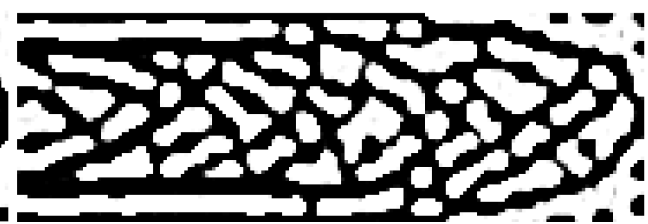

(f) Trial 6: $\left\{w_{\min }, w_{\max }\right\}=\{0.4 L, 0.1 L\}$

FIGURE 12 Cantilever beam designs with Laplacian EBs $\left[\Psi_{\ell}^{(1)}\right]$ truncated at different wavelength thresholds.

ments leveling off, however, for larger basis dimensions. The largest basis (trial 4) yields a design with only slightly better structural performance than the solution from trial 3 using hundreds fewer basis functions, suggesting that including many high-frequency EFs has diminishing benefit on improving designs. Band-pass limited designs $($ Trials 5,6$)$ recorded the largest objective values, as these structures were restricted from developing large length scale features that improve structural stiffness most; they also develop extraneous floating features, due to the restriction in the void length scale, that do not contribute to structural performance but do detract from the volume utilization.

We can confirm the low-pass and band-pass filtering induced through basis truncation by inspection of the amplitude spectra of the SFFTs of solutions 4 and 6, as shown in Fig. 13. In both cases, spectral amplitude densities are concentrated within the maximum-frequency radius induced by the wavelength-threshold criteria. Furthermore, truncation of the low index eigenfunctions, as shown in the SFFT of the Trial 6 band-pass solution (Fig. 13b), restricts the solution from containing low-frequency/large wavelength content, with the amplitude content nearly bounded between the two frequency threshold frontiers. This filtering is achieved while eliminating two linear solutions or convolutions from each objective function evaluation the low-pass PDE-filter would have required, or even potentially four linear solutions for a band-pass PDE-filter [19].

We see differing structural qualities and complexity arising from different basis truncation choices. The complexity of structural designs generally increases with EB dimension, as evidenced by the more-numerous and smaller features or holes. Structural designs obtained with these Laplacian bases $\left[\Psi_{\ell}^{(1)}\right]$, especially for lower dimensions $\ell$, tend to exhibit rounded, organic-looking features. This is caused by the relatively poor capacity of the spatially-oscillatory eigenfunctions $\psi_{j}^{(1)}$, especially those with large characteristic wavelength, to capture straighter and smaller structural members. Furthermore, though all problems were subject to the same logistic transform (Sec. 3.1) and regularization continuation schemes, the higher-dimensional designs achieve lower gray fractions (26), as the higher-frequency bases 
can capture sharper boundaries. Asymmetries in the designs likely arise from asymmetries in some basis functions; symmetric designs can be enforced, if desirable, through coefficient symmetry and suitable BCs in the EVP (12). Bandpass limited EBs develop almost cellular-looking designs, with numerous holes arising from $\beta$ being in this case sought as a linear combination of only highly oscillatory basis functions.

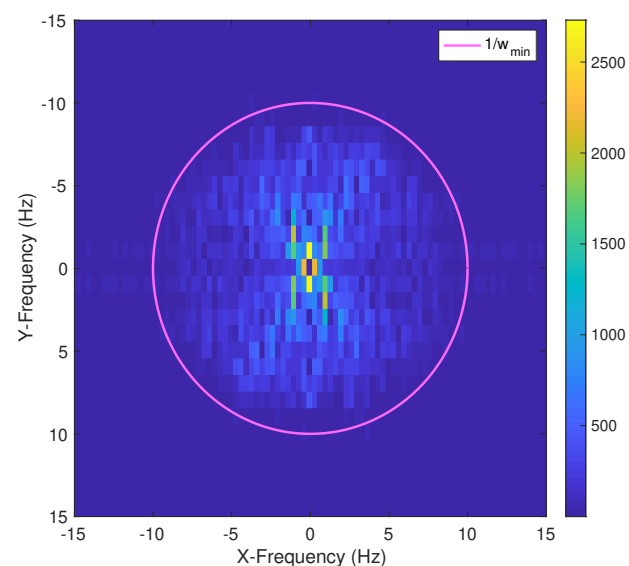

(a) SFFT of Trial 4 design

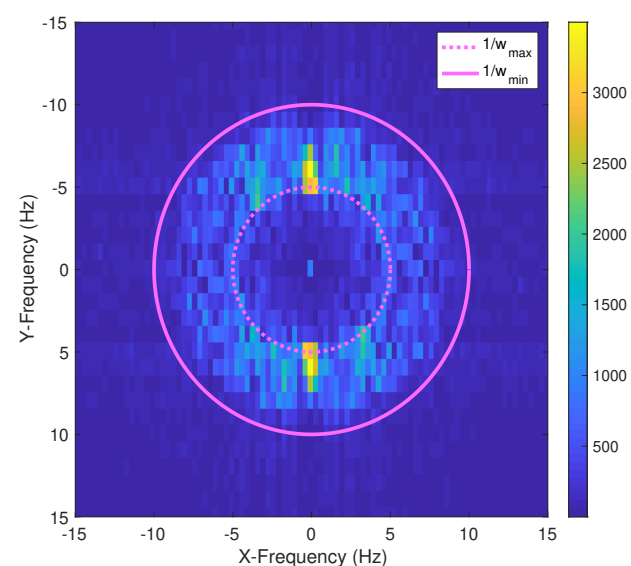

(b) SFFT of Trial 6 design

FIGURE 13 Spatial FFT of $\boldsymbol{\beta}$ fields for Trial 4 and 6 solutions; circles indicate equivalent maximum (and minimum) frequency thresholds applied through basis truncation.

\section{2 | Study: Length Scale Control}

We now turn to demonstrating the use of basis truncation as means for minimum and maximum length scale control. The chosen design configuration consists of a two-dimensional, plane-stress square structural domain $\Omega$ of side $L$ under uniform tension (Fig. 14), expecting the optimal design to be an assembly of vertical bars. We use a $80 \times 80$ bilinear FE discretization of $\Omega$, the top edge being clamped and the bottom edge experiencing a uniform, downward traction. We use again the SIMP material model (4), with the same values of void and solid phases. The continuation scheme for $\zeta$ is as in Sec. 5.1, with a maximum of 400 iterations, while $\alpha=1 \times 10^{-5}$ is kept constant. We perform three trial designs which differ by the choice of characteristic wavelength limits for band-pass basis truncation, taken as $w_{\min }, w_{\max }=\{0.2 L, 0.3 L\}$ in Trial $1, w_{\min }, w_{\max }=\{0.15 L, 0.2 L\}$ in Trial 2 and $w_{\min }, w_{\max }=\{0.1 L, 0.15 L\}$ in Trial 3.

We observe that bandpass-designed structures exhibit features with "wavelengths"-transition from void to solid consistent with the wavelengths retained in the truncated EBs. For a band-pass truncation with characteristic wavelength $\bar{w}$, we expect a design featuring approximately $1 / \bar{w}$ vertical bars. The designs yielded by the three trials conform with this expectation, as they respectively show approximately 4,6 and 8 bars (Figs. 15a, 15b and 15c). Furthermore, the resulting feature size in all three trials is approximately $\bar{w} / 2$, showing that wavelength-truncation thresholds can be set according to a desired feature size. Irregularities in the designs, such as fluctuations in the feature width or joining of parallel bars, likely occur due to the qualitative nature of the frequency filtering effected by basis truncation. Nevertheless, this example shows that truncation of the initial EB effectively allows for length scale control. 


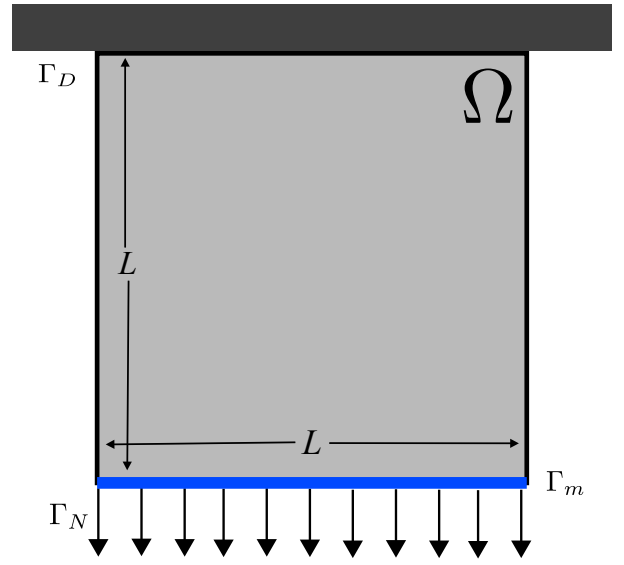

FIGURE 14 Length scale design study: structural domain under tension.

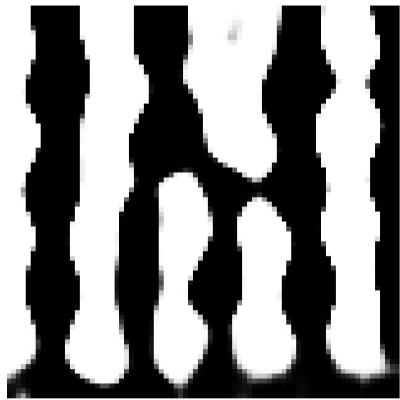

(a) Trial 1: $w_{j} \in[0.2, \hat{0} 3] \dot{L}$

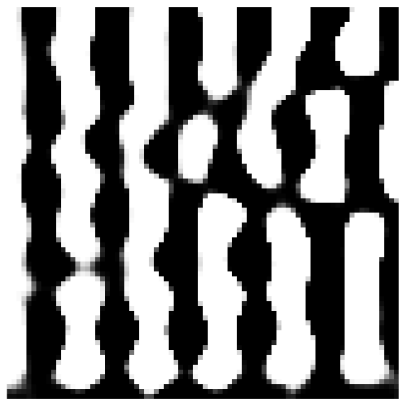

(b) Trial 2: $w_{j} \in[0.15,0.2] L$

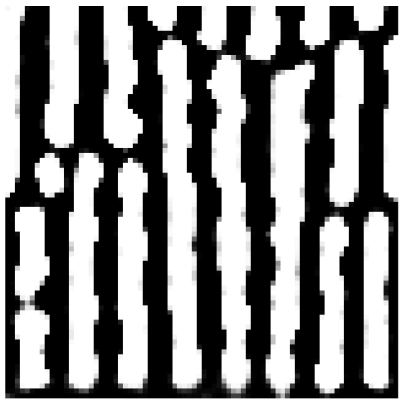

(c) Trial 3: $w_{j} \in[0.1,0.15] L$

FIGURE 15 Structures designed for tensile stiffness, using band-pass basis truncation for length scale control.

\section{3 | Study: Single versus Adapted Eigenspace Bases}

We now evaluate the performance of the basis adaptation strategy for its solution quality, basis dimension compression, and computational cost. We design the cantilever and MBB beams introduced earlier (Fig. 10b) using the proposed 4-pass AEB strategy, with the same setting of the SIMP model and void / solid phases as before, and compare results both to the 1-pass EB solution of Sec. 5.1 and results obtained with a CDM. In each case, the $3 L \times L$ rectangular structural domain $\Omega$ is modeled with $180 \times 60$ bilinear elements under plane-stress assumptions and subject to a $50 \%$ volume fraction constraint $\left(v_{\max }=0.5\right.$ in problem (18)).

We apply the 4-pass AEB method as described in Section 4.1 and Algorithms 1 and 2. We conduct two trials each for the MBB and cantilever beams, with the adapted EB dimension $\ell$ selected according to $c_{j} \leq \epsilon_{\text {tol }}$ (see (25)) with $\epsilon_{\mathrm{tol}}=0.001$ (Trial 1) or $\epsilon_{\mathrm{tol}}=0.005$ (Trial 2). Each optimization pass is solved to a gradient tolerance of $1 \times 10^{-6}$, a function decrease tolerance of $1 \times 10^{-6}$, a step-size tolerance of $1 \times 10^{-4}$, or a maximum iteration limit. We allow pass 1 to proceed for three continuation updates (maximum 300 iterations) on $\zeta$ and $\alpha$, and each subsequent pass for a maximum of 100 iterations. We employ the continuation strategy given in Sec. 5.1 for the logistic slope $\zeta$ and the 
regularization coefficient $\alpha$ (without re-initiatization between passes). We enforce a $w_{\min }=0.1 L$ lower limit on the characteristic wavelength of the eigenfunctions of Pass 1, resulting in an initial EB dimension of $\ell=700$.

To evaluate the quality of AEB solutions, we also solve the TO problems using a conventional density-based method with the same initial density $(h(\boldsymbol{\beta})=0.5)$ and under the same volume constraint. Our CDM comparison method enforces element-wise the densities $0 \leq \beta_{e} \leq 1$ and uses a Helmholtz filter with radius $r=0.0125 L$ (which provides length-scale control approximately equivalent to the AEB wavelength-threshold criterion). We use a Heaviside projection continuation scheme [14], centered at 0.5 , set with initial slope $\zeta=1$, and multiplied by 1.8 every 100 iterations. These CDM solutions are obtained using the MATLAB implementation of the MMA solver [21].

The structural solutions from the CDM and the four-pass AEB method are shown in Figure 16 for the cantilever beam and in Figure 17 for the MBB beam. Tables 2 and 4 report the final values of $\mathcal{D}(\boldsymbol{d})$ (structural objective), $f_{g}$ (gray fraction), $W$ (DWP) and the total function evaluation counts obtained for each solution. Table 3 show the structural objective values, EB dimensions and computational cost statistics from each AEB pass for the cantilever designs, while Table 5 shows those results for the MBB designs. We observe that structural objectives decrease with each successive AEB pass, as both the continuation schemes and the adapted bases enable convergence to well-defined structures with minimal intermediate gray material. Final displacement results for the trial 1 cantilever beam design (shown in Fig. $16 \mathrm{~g}$ ) outperform the best results obtained in the previous dimension-study using the single Laplacian eigenfunction basis (Cantilever AEB Trial 1: $7.32010^{2}$ vs. Cantilever Laplacian Trial 4: $7.33610^{2}$ ), under identical continuation schemes and convergence criteria and in fewer (433 vs. 471) iterations and function evaluations (448 vs 499).

Solutions obtained using the 4-pass AEB method furthermore achieve objective values within $2 \%$ of those obtained using CDMs (with the AEB-MBB Trial 1 design actually outperforming the CDM result). We obtain these structures from design spaces whose dimension $\ell$ is over two orders of magnitude smaller than that of conventional element-wise discretization of $\beta$, and about one order of magnitude smaller than that of the original Laplacian EB. AEB results are also obtained in fewer iterations and function evaluations than the CDM solution. We do note different optimization algorithms are used for the different cases and therefore exact comparison is difficult, but comparison of the number of function evaluations (and thus the number of linear solution evaluations) allows to roughly compare overall computational cost. Though the AEB strategy requires additionally the solution of four eigenvalue problems, its per-function-evaluation cost is significantly lower than the CDM: both methods require the solution of the forward and adjoint problems at each function evaluation, but the CDM requires two additional filtering linear-solutions.

\begin{tabular}{|c||c|c|c|}
\hline & AEB, Trial 1 & AEB, Trial 2 & CDM \\
\hline \hline Objective & $7.32510^{1}$ & $7.47410^{1}$ & $7.20410^{1}$ \\
\hline Gray Frac & $1.33610^{-2}$ & $1.92510^{-2}$ & $1.73510^{-2}$ \\
\hline DWP & $3.03110^{-1}$ & $3.82010^{-1}$ & - \\
\hline Total Iter & 433 & 457 & 600 \\
\hline Total Func Count & 448 & 532 & 600 \\
\hline
\end{tabular}

TAB LE 2 Final optimization results for cantilever beam designs, comparing AEB solutions to CDM results.

As reported in Table 4, both the DWP value and the basis dimensions in the adaptive passes are also consistently reduced throughout the optimization process. Decreasing DWP values signify clustering of the $\beta$ near \pm 1 values; such clustering enables effective utilization of the AEB to represent the increasingly piece-wise-constant $\beta$ field with low 


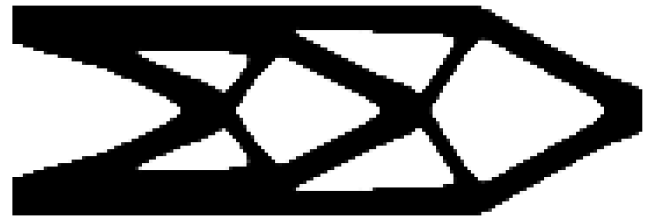

(a) CDM: Cantilever Design

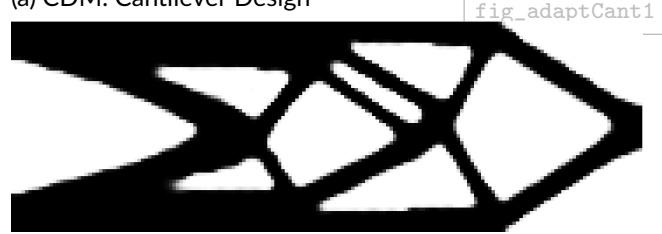

(c) AEB Trial 1: Pass 2

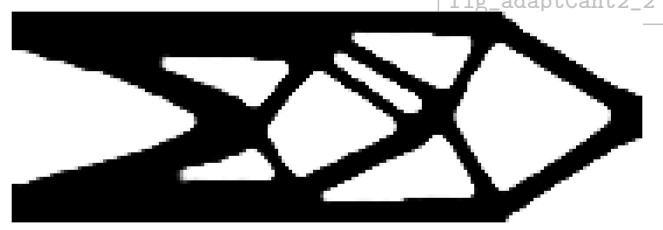

(e) AEB Trial 1: Pass 3



(g) AEB Trial 1: Pass 4

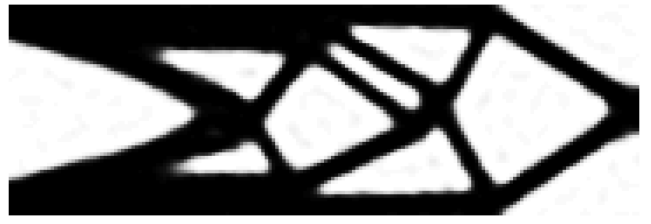

(b) AEB: Pass 1 (both trials)

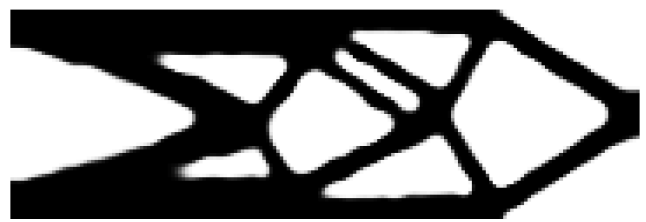

(d) AEB Trial 2: Pass 2

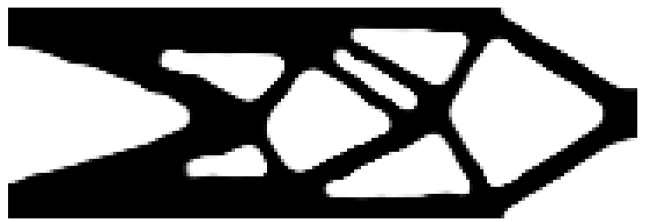

(f) AEB Trial 2: Pass 3

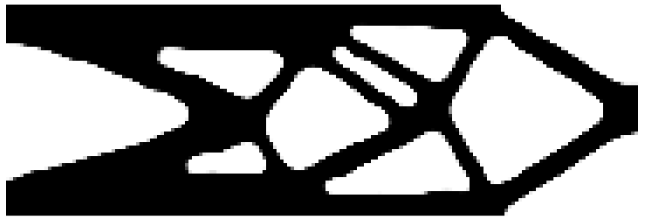

(h) AEB Trial 2: Pass 4

FIGURE 16 Cantilever beam designs produced by the 4-pass AEB method, compared with the design obtained by the CDM. Trial 1 uses $L^{2}$-error decrease tolerances of $\epsilon_{\mathrm{tol}}=10^{-3}$, Trial 2 uses $\epsilon_{\mathrm{tol}}=510^{-3}$.

dimensions. The AEB solutions yield lower final DWP evaluations than the Laplacian basis (e.g. AEB Cantilever Trial 1: $3.03110^{-1}$ vs. Laplacian Cantilever Trial 4: 1.098), reflecting the improved ability of the adapted bases to conform to piecewise-constant values. The compression is further evidenced in Figures 18a through 18d, which show the relative $L^{2}$-error achieved by projections of the final pseudo-density design from each pass onto the next adapted (reordered) basis, with increasing dimension. Error decreases quickly using the adapted bases, and more rapidly with each successive pass; for the higher projection-error tolerances, we are able to decrease the basis dimension by over an order of magnitude between passes 1 and 4 (from 700 to 23 and 31, for cantilever and MBB Trial 2 designs). We also observe that the error tolerance used strongly affects the basis dimension selected and resulting structural solution. Comparing Trial 1 and 2 results in both design scenarios, we observe tighter error tolerance yields naturally larger bases but produces improved final solutions; however, with slackened error tolerance, we trade only minor performance decreases $(\approx 2.4 \%$ for both cases) for improved final basis compression ( $>3 \times$ smaller). Final structural solutions for Trial 2 (Fig. 16h and 17h) have qualitatively identical features to the solutions obtained with the larger bases, though with slightly-rounder features.

We generally observe that solutions produced by each consecutive pass of the adaptive process to develop 


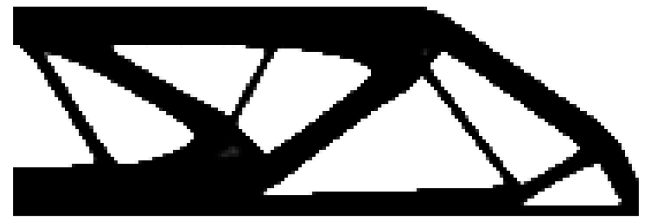

(a) CDM: MBB Design

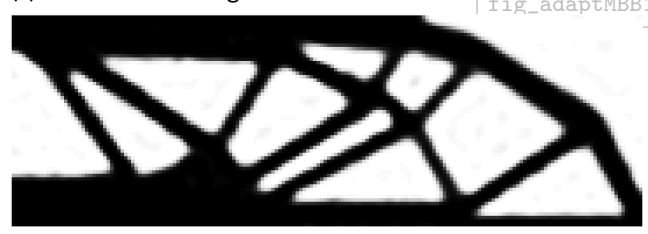

(c) AEB Trial 1: Pass 2

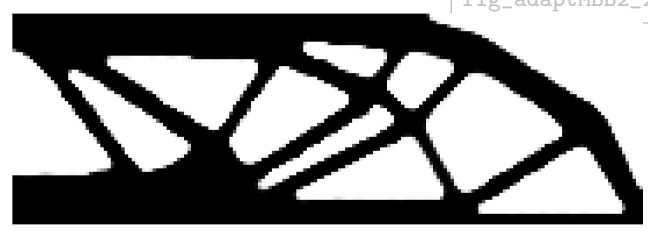

(e) AEB Trial 1: Pass 3



(g) AEB Trial 1: Pass 4

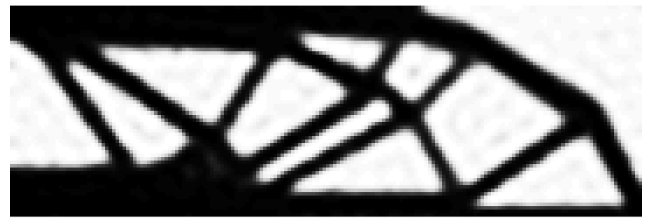

(b) AEB: Pass 1 (both trials)

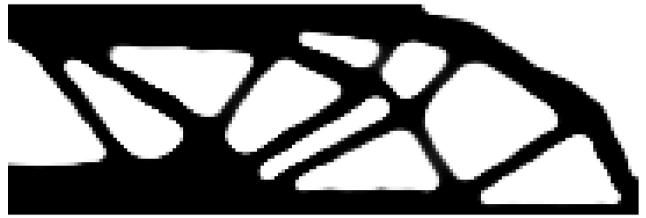

(d) AEB Trial 2: Pass 2

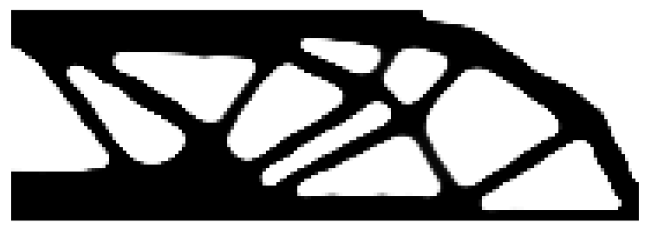

(f) AEB Trial 2: Pass 3



(h) AEB Trial 2: Pass 4

FIGURE 17 MBB beam designs produced by the 4-pass AEB method, compared with the design obtained by the CDM. Trial 1 uses $L^{2}$-error decrease tolerances of $\epsilon_{\mathrm{tol}}=10^{-3}$, Trial 2 uses $\epsilon_{\mathrm{tol}}=510^{-3}$.

sharper, smoother, and straighter features. The oscillatory character of the Laplacian EFs $\psi_{j}^{(1)}$, and the small values used for both $\zeta$ and $\alpha$ in that pass, contribute to the rounded, diffuse shape quality of Pass 1 solutions (Fig. 17b $\&$ 16b). In the adaptive bases, concentrated variation at the structural-void boundary enables refinement of the edges between void and solid. Trial 2 designs, with restricted basis dimensions, do display features with rounded or variable widths. Asymmetries in AEB solutions (e.g. Fig. $16 \mathrm{~g}$ and Fig. 16h) seemingly develop during Pass 1 due to asymmetric EFs, although provisions can be made to ensure that all basis EFs are symmetric if desired.

\section{DISCUSSION \& CONCLUSIONS}

We have presented a new design dimensionality-reduction approach for density-based topology optimization problems. Our AEB representation is an effective alternative for traditional density-based design parameterization: solutions achieve structural performance metrics on par with conventional density-based methods while allowing for drastic reduction in dimensionality of design space. It also imposes implicit regularization of the design field, providing a successful subspace in which to represent it. Representation of the fictitious density as a sum of eigenfunctions, 


\begin{tabular}{|c||c|c|c|c|}
\hline Trial 1 & Pass 1 & Pass 2 & Pass 3 & Pass 4 \\
\hline$\ell$ (EB dim.) & 700 & 383 & 159 & 84 \\
\hline Objective & $7.875810^{1}$ & $7.543310^{1}$ & $7.394310^{1}$ & $7.325810^{1}$ \\
\hline DWP & 4.128 & 1.238 & $4.43310^{-1}$ & $3.03110^{-1}$ \\
\hline Iter Count & 261 & 68 & 50 & 54 \\
\hline Func Count & 271 & 71 & 51 & 55 \\
\hline
\end{tabular}

\begin{tabular}{|c||c|c|c|c|}
\hline Trial 2 & Pass 1 & Pass 2 & Pass 3 & Pass 4 \\
\hline$\ell$ (EB dim.) & 700 & 140 & 47 & 23 \\
\hline Objective & $7.875810^{1}$ & $7.714010^{1}$ & $7.558510^{1}$ & $7.474110^{1}$ \\
\hline DWP & 4.128 & 1.486 & $6.25210^{-1}$ & $3.82010^{-1}$ \\
\hline Iter Count & 261 & 41 & 99 & 56 \\
\hline Func Count & 271 & 42 & 146 & 73 \\
\hline
\end{tabular}

TAB LE 3 Cantilever: design results for successive AEB passes, trial 1 (top) and trial 2 (bottom).

\begin{tabular}{|c||c|c|c|}
\hline & AEB, Trial 1 & AEB, Trial 2 & CDM \\
\hline Objective & $1.196010^{2}$ & $1.228110^{2}$ & $1.20010^{2}$ \\
\hline Gray Frac & $1.57810^{-2}$ & $2.486210^{-2}$ & $1.70310^{-2}$ \\
\hline DWP & $3.48710^{-1}$ & $5.164110^{-1}$ & - \\
\hline Total Iter & 382 & 426 & 600 \\
\hline Total Func Count & 433 & 446 & 600 \\
\hline
\end{tabular}

TAB LE 4 Final optimization results for MBB beam designs, comparing AEB solutions to CDM results.

and its transformation under the logistic function, removes the computational expense of element-wise 0-1 bound constraints and density-filtering schemes. Convergence to finite basis coefficients is secured through regularization by means of a double-well potential, which promotes convergence of $\beta$ to \pm 1 (i.e. finite) values.

Through the adaptive strategy proposed, the design basis is updated to represent the current solution trajectory, enabling further compression of the design space and acceleration of the optimization solution. Meanwhile, the double-well potential regularization encourages this effective compression, as the fictitious-density solutions are steered towards bimodal, piecewise constant fields which may be approximated in low-dimensional form using the adapted eigenfunctions. The four-stage adaptive sequence proposed works well to reduce basis dimensionality iteratively and ultimately obtain good-quality structural solutions; further basis compression may be obtained through additional, or more frequent, adaptive passes. The resulting small-dimensional design space enables our use of optimization algorithms (the primal-dual interior-point method) typically impractical in conventional TO problems due to 


\begin{tabular}{|c||c|c|c|c|}
\hline Trial 1 & Pass 1 & Pass 2 & Pass 3 & Pass 4 \\
\hline$\ell$ (EB dim.) & 700 & 435 & 175 & 93 \\
\hline Objective & $1.355010^{2}$ & $1.249610^{2}$ & $1.209710^{2}$ & $1.196010^{2}$ \\
\hline DWP & 1.276 & $6.31510^{-1}$ & $4.01010^{-1}$ & $3.48710^{-1}$ \\
\hline Total Iter & 235 & 59 & 44 & 44 \\
\hline Total Func Count & 247 & 60 & 54 & 60 \\
\hline
\end{tabular}

\begin{tabular}{|c||c|c|c|c|}
\hline Trial 2 & Pass 1 & Pass 2 & Pass 3 & Pass 4 \\
\hline$\ell$ (EB dim.) & 700 & 189 & 61 & 31 \\
\hline Objective & $1.355010^{2}$ & $1.277610^{2}$ & $1.244110^{2}$ & $1.228110^{2}$ \\
\hline DWP & 1.276 & 1.049 & $6.87110^{-1}$ & $5.16410^{-1}$ \\
\hline Iter Count & 235 & 68 & 63 & 55 \\
\hline Func Count & 247 & 70 & 73 & 56 \\
\hline
\end{tabular}

TAB LE 5 MBB beam: design results for successive AEB passes, trial 1 (top) and trial 2 (bottom).

their high dimensionality. For example, the primal-dual interior point method in MATLAB requires the factorization of a $n_{\text {var }} \times n_{\text {var }}$ Hessian-approximation to compute design step updates, where $n_{v a r}$ is the number of design variables [20], in which case the moderate AEB dimension is clearly favorable.

An additional, unique benefit of the EB truncation is its resulting implicit control over solution length scale. We have shown that truncation of the basis produced by Pass 1 operates with similar effect to either low-pass or bandpass filter operations, and thus operates as means of controlling solution length scale. We see from these results, and in our general experience, that structural topology is generally defined in the first optimization pass, as the initial solution using the Laplacian EB determines the general solution form, including the size and numbers of structural features. The adapted bases are adept at capturing and perturbing the current solution, though may not be equipped to represent dramatically different structural forms. New features may still evolve and old features disappear during the adapted passes, but drastic shifts in solution character are unlikely during the later adaptive passes. We also note that band-pass length-scale control, especially restriction of the solution to high frequency content, is not always preserved in subsequent passes; a solution featuring only small-scale features, for example, may redevelop larger features once the adapted bases are used. Further study is needed to maintain band-pass length scale control in the adaptive passes.

Several additional questions may be pursued to maximize the utility of the AEB method. We have not established explicit uniqueness or mesh-independence of solutions, as designs using the same dimension $A E B$, but with different mesh discretization or eigenfunction members, can converge to qualitatively different designs. This behavior likely owes to slight numerical differences in the eigenfunctions computed for different meshes, so that designs on different meshes are effectively confined to different eigenfunction subspaces. However, constraint of the spatial variation wavelength to a given level does generally result in qualitatively similar structural solutions. Next, as the AEB method provides global, rather than element-wise, parameterization of the design field, local element-wise constraints are not applicable. Thus, methods that impose local constraints or operations, including strict length-scale, 


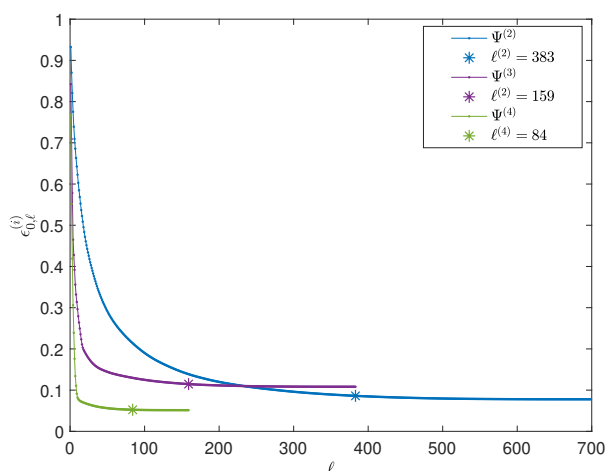

(a) Cantilever Design: Trial 1

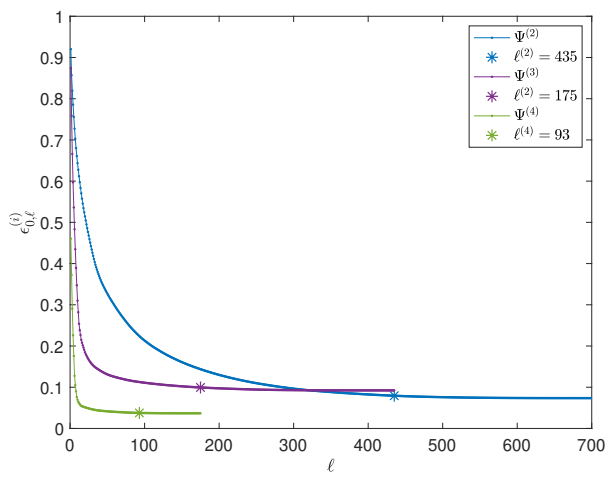

(c) MBB Design: Trial 1

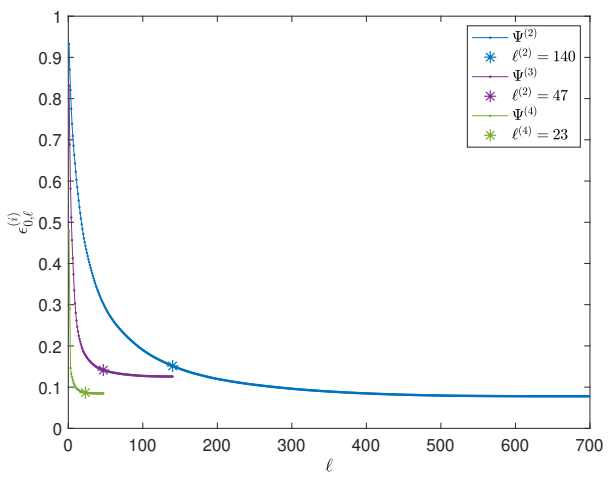

(b) Cantilever Design: Trial 2

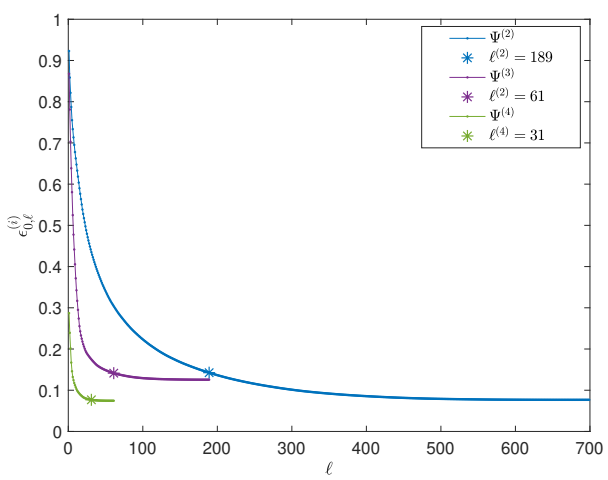

(d) MBB design: Trial 2

FIGURE 18 Relative $L^{2}$ residuals $\epsilon_{0, \ell}^{(i)}$, computed with bases of increasing dimension $\ell$. Stars indicate basis dimension selected. Increased error tolerances in Trial 2 examples allow for decreased dimension selections.

such as morphological filters (e.g. [23]) or maximum-overhang (e.g. [24]) onto TO designs need redevelopment in the AEB context.

Additional methods to improve the computational performance of the AEB method may likewise be pursued. Sparse storage of the eigenfunctions may be an attractive method to reduce memory costs and implement the method in parallel, as suggested in [8]. As higher-index eigenfunctions have only localized regions of variation and near-zero values elsewhere, EF's may reasonably be stored in a sparse format, with only non-zero values and associated elementindices. Such storage format would save memory-costs in a parallelized, domain decomposed implementation. Mesh adaptivity schemes have been explored for other adaptive eigenspace inversion formulations [10] and would prove useful in the present framework for improved refinement of boundaries between material phases. Next, the use of ubiquitous MMA solvers for TO problems requires additional care for the AEB formulation, primarily because users must supply design variable bounds to initialize the solver. Initial steps in MMA solvers risk pushing the basis coefficients associated with high-frequency eigenfunctions to the supplied bounds, causing premature convergence to nearly-random, black-white patterns. Aggressive continuation strategies and tailored eigenfunction coefficient bounds are necessary for successful implementation of the AEB method with MMA solvers. 
We view the AEB method as a promising alternative to conventional element-wise parameterizations of the design field. The method's main benefits are its significant design dimensionality reduction, its replacement of conventional density filtering operations, and its implementation of simple length scale control. Operation of the AEB method with

a mesh-adaptation scheme will further emphasize the improved resolution of the eigenfunction variation near large density gradient regions. We predict the AEB method, whose essential characteristics do not depend on the specific physics considered, may be useful to topology optimization in many physical contexts.

\section{7 | ACKNOWLEDGEMENTS}

Support from the US Department of Energy Computational Science Graduate Fellowship, provided through grant DE-FG02-97ER25308, is gratefully acknowledged. We thank Dr. Krister Svanberg for providing the MATLAB implementation of the Globally Convergent Method of Moving Asymptotes, used to generate the results in this work.

\section{A I IMPLEMENTATION DETAILS}

In this appendix, we discuss a few details related to operation of the AEB method for topology optimization.

\section{A.1 | Continuation Schemes \& Effects of Double-Well Potential Term}

In our formulation, convergence to successful black-white designs depends on an interplay between the logistic slope parameter $\zeta$ and the regularization coefficient $\alpha$. The combination of the logistic transform and the DWP regularization ensures that designs satisfy minimum standards, namely that (a) analysis densities reside in [0,1] and (b) EB coefficient solutions do not approach unbounded values. However, the combination of $\zeta$ and $\alpha$ will strongly affect the convergence to good quality solutions. Continuation strategies are ultimately useful to control convergence of the solution to optimal, near 0-1 analysis density designs.

A successful continuation strategy for the AEB method initializes $\zeta$ and $\alpha$ at small values (e.g. $\zeta=2, \alpha_{0}=10^{-4}$ ), then gradually increases them. The initial development of an advantageous topology pattern - albeit still with intermediate densities - can permit subsequent basis adaptations to emphasize these developing structural-void boundaries and obtain high-quality solutions in low-dimensional form. We find that only modest increase factors of the DWP weighting are necessary to provide the desired bimodal clustering; continuation of the slope parameter to large values $(\zeta>10)$ is more important since we want analysis densities $h(\beta)$ at $\beta= \pm 1$ to be sufficiently close to 0 or 1 . Though we have not established explicit criteria for selection of the DWP weighting coefficient, it appears to be connected to the overall lengthscale desired for the structure.

\section{A.2 | Algorithm Selection}

We may solve the PDE-constrained design problem using standard nonlinear programming algorithms. AEB design studies in this report use an interior-point method as implemented in MATLAB fmincon, choosing a full-step strategy and assigning KKT-norm, step-norm, and function-step convergence criteria [20]. The EVP (12) is computed with the MATLAB eigenvalue solver, which uses Lanczos iteration and determines the eigenvectors associated with a lowest subset of eigenvalues of the discretized EVP. We note the AEB formulation may alternatively use the Method of Moving Asymptotes (MMA) method, the popular algorithmic option for solving constrained minimization problems 
in density-based TO [21]. However, our formulation does pose some issues for MMA solvers. Successful operation of the MMA optimizer using the AEB approach requires user-supplied, initial artificial design variable bounds. We do not have a priori estimates of the values (or magnitudes) the basis coefficients should take, but we may provide artificial design bounds in reference to the values from an initial eigenfunction coefficient guess, for example. Larger regularization coefficients may also be useful to avoid immediate convergence to random, 0-1 solutions.

\section{references}

[1] Sigmund O, Petersson J. Numerical instabilities in topology optimization: a survey on procedures dealing with checkerboards, mesh-dependencies and local minima. Structural optimization 1998;16(1):68-75.

[2] Bourdin B. Filters in topology optimization. International journal for numerical methods in engineering 2001;50(9):21432158.

[3] Lazarov BS, Sigmund O. Filters in topology optimization based on Helmholtz-type differential equations. International Journal for Numerical Methods in Engineering 2011;86(6):765-781.

[4] Guest JK, Smith Genut LC. Reducing dimensionality in topology optimization using adaptive design variable fields. International journal for numerical methods in engineering 2010;81(8):1019-1045.

[5] Huang W, Sun W. Variational mesh adaptation II: error estimates and monitor functions. Journal of Computational Physics 2003;184(2):619-648.

[6] Lambe AB, Czekanski A. Topology optimization using a continuous density field and adaptive mesh refinement. International Journal for Numerical Methods in Engineering 2018;113(3):357-373.

[7] Wang Y, Kang Z, He Q. Adaptive topology optimization with independent error control for separated displacement and density fields. Computers \& Structures 2014;135:50-61.

[8] Grote MJ, Kray M, Nahum U. Adaptive eigenspace method for inverse scattering problems in the frequency domain. Inverse Problems 2017;33(2):025006.

[9] Grote MJ, Nahum U. Adaptive eigenspace for multi-parameter inverse scattering problems. Computers \& Mathematics with Applications 2019;77(12):3264-3280.

[10] De Buhan M, Darbas M. Numerical resolution of an electromagnetic inverse medium problem at fixed frequency. Computers \& Mathematics with Applications 2017;74(12):3111-3128.

[11] Baffet $\mathrm{DH}$, Grote $\mathrm{MJ}$, Nahum $\mathrm{U}$, Adaptive eigenspace method for inverse scattering problems in the frequency domain; 2020. Preprint in preparation.

[12] Bendsøe MP. Optimal shape design as a material distribution problem. Structural optimization 1989;1(4):193-202.

[13] Wang F, Lazarov BS, Sigmund O. On projection methods, convergence and robust formulations in topology optimization. Structural and Multidisciplinary Optimization 2011;43(6):767-784.

[14] Xu S, Cai Y, Cheng G. Volume preserving nonlinear density filter based on heaviside functions. Structural and Multidisciplinary Optimization 2010;41(4):495-505.

[15] Hinze M, Pinnau R, Ulbrich M, Ulbrich S. Optimization with PDE constraints, vol. 23. Springer Science \& Business Media; 2008.

[16] Guest JK, Asadpoure A, Ha SH. Eliminating beta-continuation from heaviside projection and density filter algorithms. Structural and Multidisciplinary Optimization 2011;44(4):443-453.

[17] Burger M, Stainko R. Phase-field relaxation of topology optimization with local stress constraints. SIAM Journal on Control and Optimization 2006;45(4):1447-1466.

[18] Strong D, Chan T. Edge-preserving and scale-dependent properties of total variation regularization. Inverse problems 2003;19(6):S165.

[19] Lazarov BS, Wang F. Maximum length scale in density based topology optimization. Computer Methods in Applied Mechanics and Engineering 2017;318:826-844.

[20] Constrained Nonlinear Optimization Algorithms - MATLAB \& Simulink;. https://www . mathworks . com/help/optim/ug/ constrained-nonlinear-optimization-algorithms.html.

[21] Svanberg K. The method of moving asymptotes-a new method for structural optimization. International journal for 
numerical methods in engineering 1987;24(2):359-373.

[22] Sigmund O. Morphology-based black and white filters for topology optimization. Structural and Multidisciplinary Optimization 2007;33(4-5):401-424.

[23] Zhou M, Lazarov BS, Wang F, Sigmund O. Minimum length scale in topology optimization by geometric constraints. Computer Methods in Applied Mechanics and Engineering 2015;293:266-282.

[24] Gaynor AT, Guest JK. Topology optimization considering overhang constraints: Eliminating sacrificial support material in additive manufacturing through design. Structural and Multidisciplinary Optimization 2016;54(5):1157-1172. 